Federal Reserve Bank of Minneapolis

Research Department Staff Report 281

November 2000

\title{
Technology (and Policy) Shocks in Models of Endogenous Growth ${ }^{*}$
}

\author{
Larry E. Jones \\ Federal Reserve Bank of Minneapolis \\ and University of Minnesota
}

Rodolfo E. Manuelli

University of Wisconsin

Ennio Stacchetti

University of Michigan

\begin{abstract}
Our objective is to understand how fundamental uncertainty can affect the long-run growth rate and what factors determine the nature of the relationship. Qualitatively, we show that the relationship between volatility in fundamentals and policies and mean growth can be either positive or negative. We identify the curvature of the utility function as a key parameter that determines the sign of the relationship. Quantitatively, we find that when we move from a world of perfect certainty to one with uncertainty that resembles the average uncertainty in a large sample of countries, growth rates increase, but not enough to account for the large differences in mean growth rates observed in the data. However, we find that differences in the curvature of preferences have substantial effects on the estimated variability of stationary objects like the consumption/output ratio and hours worked.

*We thank Larry Christiano, Martin Eichenbaum, Fernando Alvarez, and Craig Burnside for their help, Henry Siu for assistance, and the National Science Foundation for financial support. The views expressed herein are those of the authors and not necessarily those of the Federal Reserve Bank of Minneapolis or the Federal Reserve System.
\end{abstract}




\section{Introduction}

In his celebrated 1987 book, Models of Business Cycles, Robert Lucas presents some simple calculations to argue that the trade-off between fluctuations and growth is such that a representative agent's willingness to pay, in terms of growth rates, for a more stable environment is almost zero. Even though Lucas' conclusion has been challenged by analyses of models that relax some of the details in his basic environment-ranging from the specification of preferences to the details of the market structure $^{1}$-none of these analyses question a fundamental implicit assumption: the factors that affect fluctuations do not affect long-run growth. ${ }^{2}$

Is there any evidence that the volatility of shocks, both policy and productivity shocks, has an impact on long-run growth? The results are mixed. In an early study, Kormendi and Meguire (1985) find that variability in output is positively related to mean growth in a cross section of countries. More recently, Ramey and Ramey (1995) find that higher volatility decreases growth, also in a cross section of countries. Empirical work that relates policy variability, mostly inflation, and growth also seems to point to a negative relationship (Judson and Orphanides 1996). Simple regressions of mean growth rates on measures of volatility of growth rates suggest a U-shaped relationship, with an upward sloping segment only at very high levels of volatility.

\footnotetext{
${ }^{1}$ For the former, see Manuelli and Sargent (1988); for the latter, Imrohoroğlu (1989) and Atkeson and Phelan (1994).

2 The current standard in the real business cycle literature is to view long-run growth as exogenous and, hence, independent of the fundamental shocks. For an explicit discussion, see Cooley and Prescott (1995).
} 
Our objective in this paper is to study a class of models in the neoclassical tradition in which fundamental uncertainty can affect the long-run growth rate. ${ }^{3}$ Our main focus is to understand both qualitatively and quantitatively how important fluctuations are for growth. Our analysis includes both theoretical results and numerical evaluations.

Qualitatively, we show that the relationship between volatility in fundamentals and policies and mean growth can be either positive or negative. For the case of independent and identically distributed driving shocks—even allowing for endogenously determined rates of return—our results are in line with those found in related models: if preferences have curvature at least as high as the $\log$, increased volatility increases growth, while for low curvature, the opposite is true. ${ }^{4}$ We then show how the negative relationship between volatility and growth also holds—for the case in which curvature is at least as high as the log-for economies with correlated shocks. Overall, we expect a positive relationship between uncertainty and growth. Thus, for a class of simple specifications, elimination of the type of fluctuations we study will decrease long-run growth.

We also find that the decomposition of the variance of the fundamental shocks into its autocorrelation and innovation variance components matters: increases in the variability of the innovations to the fundamental shocks are likely to have a larger impact than increases in the serial correlation. Finally, we show that these models can generate positively autocorrelated growth rates, but for this to be the case, the driving shocks must be positively autocorrelated themselves.

${ }^{3}$ Although we emphasize a technology-shock interpretation of the type used in the real business cycle literature for the shocks in our model (see Cooley 1995 for an up-to-date survey of this literature), the shocks that we model can also be interpreted as random fiscal policies (for an equivalence result, see Jones and Manuelli 1999).

${ }^{4}$ Following the work of Eaton (1981), Obstfeld (1994) studies the case of two linear technologies which, in fact, makes the rate of return exogenous. More recently, de Hek (1999) analyzes a case in which an externality gives rise to linear $A k$ technology in equilibrium, and he finds that log utility is the critical specification. 
Even though our work follows the recent analyses of stochastic endogenous growth models in which the source of shocks is technology, ${ }^{5}$ policies, ${ }^{6}$ or a combination of the two, ${ }^{7}$ it has a different emphasis. Our main point is to highlight the critical roles played by the degree of risk aversion and the nature of the stochastic process for the exogenous uncertainty on the distribution of growth rates.

To explore the quantitative importance of uncertainty for growth, we simulate a general version of our model. The numerical exercise is revealing. The main result is that when we move from a world of perfect certainty to one with uncertainty that resembles the average uncertainty in a large sample of countries, growth rates increase somewhere between 0.17 percent and 0.80 percent, with 0.20 percent being a reasonable estimate. Even though these are nontrivial changes, they are not large enough by themselves to account for the large differences in mean growth rates observed in the data.

As expected, differences in the curvature of the utility function and the specification of the fundamental uncertainty have impacts on the second-order properties of growth rates. In general, increases in the coefficient of risk aversion decrease the standard deviation of the growth rate, and increases in the serial correlation of the exogenous shocks also increase the serial correlation of the growth rate. Unlike exogenous growth models, the class of models we study can generate positively autocorrelated growth rates.

${ }^{5}$ For example, see King, Plosser, and Rebelo (1988), King and Rebelo (1988), Obstfeld (1994), and de Hek (1999).

${ }^{6}$ See Eaton (1981), Bean (1990), Aizenman and Marion (1993), Gomme (1993), Hopenhayn and Muniagurria (1996), and Dotsey and Sarte (1997).

${ }^{7}$ See, for example, Kocherlakota and Yi (1997). 
Differences in the curvature of preferences have substantial effects on the estimated variability of stationary objects like the consumption/output ratio and hours worked. For this reason, we expect that the models considered in this paper will provide the basis for sharp estimates of the curvature parameter. This is in contrast to the results in exogenous growth models in which curvature has only a small effect and confirms the critical role that the shape of the utility function has in determining the long-run growth rate in nonstochastic versions of models of endogenous growth.

Section 2 presents the basic analytic results. Section 3 discusses a key property of endogenous growth models that makes them computationally tractable. Section 4 contains numerical results for calibrated versions of the model, and Section 5 offers some concluding comments.

\section{Stochastic Growth Models: Analytic Results}

In this section, we explore the theoretical implications of increases in uncertainty on the distribution of growth and savings rates. Not surprisingly, an analytic characterization of the solutions to stochastic endogenous growth models is hard to come by. For this reason, we will restrict attention to two simple but revealing examples. In the first, we explore the effects of i.i.d. shocks with an elastic labor supply. The second example is a version of the $A k$ model (alternatively, the labor supply is exogenous), but allows for correlated shocks. Our results are extensions of the literature on optimal savings (for example, Phelps 1962, Levhari and Srinivasan 1969, and Rothschild and Stiglitz 1971) expanded to incorporate general equilibrium effects, an elastic labor supply, and serially correlated shocks. ${ }^{8}$

\footnotetext{
${ }^{8}$ Eaton (1981) was the first to apply these ideas to growth models. A two-technology version is in Obstfeld (1994).
} 
The equilibrium of the class of models we study can be computed as the solution to the following planner's problem:

(P.1) $\max E\left\{\sum_{t} c_{t}^{1-\sigma} v\left(n_{t}\right) /(1-\sigma)\right\}$

subject to

$$
c_{t}+k_{t+1}+h_{t+1} \leq s_{t} A k_{t}^{\alpha}\left(n_{t} h_{t}\right)^{1-\alpha}+(1-\delta) k_{t}+(1-\delta) h_{t}
$$

$h_{0}$ and $k_{0}$ given. The shock process $\left\{s_{t}\right\}$ is assumed to be a linear Markov process given by

$$
s_{t+1}=1-\rho+\rho s_{t}+\varepsilon_{t+1}
$$

where $\varepsilon_{t+1}$ is white noise. We assume that the distribution of $\varepsilon$ is given by the measure $\mu_{\theta}$, where $\theta$ is an index of riskiness. More precisely, $\theta^{\prime}>\theta$ means that $\mu_{\theta^{\prime}}$ is dominated in the sense of secondorder stochastic dominance by $\mu_{\theta}$. Thus, a higher $\theta$ corresponds to higher volatility of the innovation to the technology shock. For this specification, the standard deviation of the $\left\{s_{t}\right\}$ process (using the invariant distribution) is given by $\sigma_{s}=\sigma_{\varepsilon} /\left(1-\rho^{2}\right)^{1 / 2}$, where $\sigma_{\varepsilon}$ is the standard deviation of the innovation. If we define increases in $\sigma_{s}$, holding the mean constant at one, as increases in risk, it follows that there are two ways to increase risk: increase $\sigma_{\varepsilon}$, and increase $\rho$. In our setting, increases in $\theta$ correspond to increases in the variability of the innovations, $\sigma_{\varepsilon}$. Even though we will take $\left\{s_{t}\right\}$ to be a productivity shock, we can reinterpret $1-s_{t}$ as a tax shock, provided that income is used to buy a good that does not affect preferences for consumption and leisure. ${ }^{9}$

We assume that $v$ is such that the utility function is concave and that the marginal utility of working is negative.

\footnotetext{
${ }^{9}$ For a more thorough discussion, see Jones and Manuelli (1999). Dotsey and Sarte (1997) also mention the connection between monetary shocks and tax shocks.
} 


\subsection{The i.i.d. Case}

In our first example, we consider i.i.d. shocks $(\rho=0)$ and full depreciation of both stocks $(\delta=1)$. To guarantee that an equilibrium exists, the economy cannot be too productive (for a discussion, see Jones and Manuelli 1990). For this example, the relevant condition, which we assume holds, is

$$
\left[\beta\left(A(1-\alpha)^{(1-\alpha)} \alpha^{\alpha}\right)^{(1-\sigma)}\right]^{1 / \sigma}\left(\int_{S}(1+\varepsilon)^{(1-\sigma)} \mu_{\theta}(d \varepsilon)\right)^{1 / \sigma}<1
$$

To ensure an interior (in terms of $n$ ) solution, we need stronger conditions, namely,

(C.1) $\left[\beta\left(A(1-\alpha)^{(1-\alpha)} \alpha^{\alpha}\right)^{(1-\sigma)}\right]^{1 / \sigma}\left(\int_{S}(1+\varepsilon)^{(1-\sigma)} \mu_{\theta}(d \varepsilon)\right)^{1 / \sigma}<1-\left[(\sigma-1)(1-\alpha) v(1) / v^{\prime}(1)\right]$

and

(C.2) if $0<\sigma<1, \lim _{n \rightarrow 0} 1-\left[(\sigma-1)(1-\alpha) v(n) /\left(n v^{\prime}(n)\right)\right]<0$.

These two conditions guarantee that the equilibrium labor supply is strictly between 0 and 1. We assume that both conditions hold. From now on, we will describe the conditions for the case in which $\sigma \neq 1 .^{10}$

We next argue that the equilibrium decision rules display three properties: saving is a constant fraction, $\varphi$, of income; the labor supply is constant; and the ex post rates of return to physical and human capital are equal. First, if rates of return to the two forms of capital are equal (for each realization of $s$ ) then the stocks of human and physical capital must satisfy $h_{t}=[(1-\alpha) / \alpha] k_{t}$. Given this, the saving rate, $\varphi$, and the level of employment, $n$, must solve

$$
\begin{aligned}
& \varphi=1-\left[(\sigma-1)(1-\alpha) v(n) /\left(n v^{\prime}(n)\right)\right] \\
& \varphi=D \hat{s}^{1 / \sigma} n^{(1-\alpha)(1-\sigma) / \sigma}
\end{aligned}
$$

${ }^{10}$ If $\sigma=1$, the utility function is $\log (c)+v(n)$, with $v^{\prime}(n)<0$. In this case, conditions (C.1) and (C.2) take slightly different forms. We present the derivation in the proof of Proposition 1. 
where $A^{*}$ is $A(1-\alpha)^{(1-\alpha)} \alpha^{\alpha}, D=\left[\beta\left(A^{*}\right)^{(1-\sigma)}\right]^{1 / \sigma}$, and $\hat{s}=\int_{S}(1+\varepsilon)^{(1-\sigma)} \mu_{\theta}(d \varepsilon)$. Basically, equation (2.1) guarantees that at the conjectured equilibrium, the marginal rate of substitution between consumption and income is equal to the real wage, while (2.2) is the Euler equation that ensures equality between the intertemporal marginal rate of substitution in consumption and the rate of return on capital. Let the solution to (2.1) and (2.2) be a pair $(\varphi, n)$, which depends on the parameters $\left(\sigma, \mu_{\theta}\right)$. An equilibrium is fully characterized by this pair. The growth rate associated with this equilibrium is given by (2.3) $y_{t+1} / y_{t} \equiv \gamma_{t+1}=\mathrm{s}_{t+1} A^{*} n^{1-\alpha} \varphi=s_{t+1} \gamma$

where, since $E s_{t}=1, \gamma$ is the mean growth rate.

Proposition 1: Assume that conditions (C.1) and (C.2) hold. Then an equilibrium of the conjectured form exists and is unique. Moreover, if $\theta^{\prime}>\theta$, the equilibrium satisfies the following conditions:

(a) The effects of increases in risk:

(i) $(\varphi, n, \gamma)$ increase with $\theta$ if $\sigma>1$.

(ii) $(\varphi, n, \gamma)$ decrease with $\theta$ if $0<\sigma<1$.

(iii) $(\varphi, n, \gamma)$ are independent of $\theta$ if $\sigma=1$.

(b) Amplification: The ratio of the standard deviation of the growth rate to the standard deviation of the technology shock, $\sigma_{\gamma} / \sigma_{s}$, satisfies the following:

(i) $\sigma_{\gamma} / \sigma_{s}>1$ if the growth rate is positive $(\gamma>1)$.

(ii) $\sigma_{\gamma} / \sigma_{s}$ increases with $\theta$ if $\sigma>1$.

(iii) $\sigma_{\gamma} / \sigma_{s}$ decreases with $\theta$ if $0<\sigma<1$.

(iv) $\sigma_{\gamma} / \sigma_{s}$ is independent of $\theta$ if $\sigma=1$.

Proof: See Appendix A. 
Thus, in very simple economies, it is clear that even the sign of the effect of increased uncertainty on average growth rates varies with preference parameters. The model can accommodate a positive relationship, consistent with the findings of Kormendi and Meguire (1985), if $\sigma>1$, or a negative relationship, as found by Ramey and Ramey (1995), if $0<\sigma<1 .{ }^{11}$ The i.i.d. (and full depreciation) version of the model has a sharp implication about a second moment of the distribution of growth rates: the first-order serial correlation coefficient is zero. This statistic has been at the center of the arguments on the inability of the real business cycle model to display persistence (see Cogley and Nason 1995 and Hall 1998). The most-cited evidence for nonzero serial correlation is described by Cogley and Nason (1995). These authors find a small positive autocorrelation in annual data for the United States. ${ }^{12}$ Fatas (2000) also finds that for a group of 15 developed countries, the growth rates are autocorrelated. The international evidence, using the full Summers-Heston (1991, 1993) data set at annual frequencies, is less conclusive. In a sample of 148 countries, we find that in more than two-thirds of the countries, the point estimate of the first-order autocorrelation coefficient is not significantly different from zero. ${ }^{13}$ In the majority, but not all, of the remaining countries, the point estimate is greater than zero and the average of the point estimates is close to 0.3 . Thus, the evidence suggests that there is a fair amount of heterogeneity in the distribution of the serial correlation coefficients of the growth rate across countries.

\footnotetext{
${ }^{11}$ There is one borderline case in which changes in $\theta$ have no impact on growth rates. This case corresponds to $v(n)=n^{1+\chi} /(1+\chi)$, with $(1+\chi)>0$ and $\sigma>1$. For this specification $v^{\prime}(n) n / v(n)$ is constant and $(2.1)$ pins down $\varphi$, which, of course, is independent of the properties of $\left\{s_{t}\right\}$.

${ }^{12}$ However, when the Summers-Heston data set for the United States is used, it is not possible to reject the hypothesis that the annual first-order correlation coefficient is zero.

${ }^{13}$ We used $t$-statistics in excess of 1.4 as indicating rejections of the null hypotheses of zero autocorrelation. Had we used the more stringent threshold of 2.5 , we would have rejected the null for only ten countries, which is within the standard margin.
} 


\subsection{The Markov Case}

Our next step is to show how properties of the stochastic process $\left\{s_{t}\right\}$ and the rate of depreciation affect the distribution of growth rates. To this end, we study a version of problem (P.1) which abstracts from labor supply—we set $v(n)$ equal to one—but allows for both serially correlated shocks $(\rho \neq 0)$ and less than full depreciation $(\delta<1)$. It turns out that both elements are important.

In order to guarantee that the solution to the planner's problem, (P.1), is well defined, it is necessary to bound how fast output can grow in this economy. A natural generalization of the condition discussed in Jones and Manuelli (1990) is

$\sup _{s} \beta \int\left(A\left(1-\rho^{+} \rho s\right)+1-\delta+\varepsilon\right)^{1-\sigma} \mu_{\theta}(d \varepsilon) \leq \kappa<1$.

In Proposition 2, we show that the optimal capital accumulation rule can be written as

$k_{t+1}=\varphi\left(s_{t} ; \theta, \rho\right)\left(s_{t} A+1-\delta\right) k_{t}$.

In this case, the growth rate is given by

$$
y_{t+1} / y_{t} \equiv \gamma\left(s_{t}, s_{t+1} ; \theta, \rho\right)=s_{t+1} \varphi\left(s_{t} ; \theta, \rho\right)\left[s_{t} A+1-\delta\right] / s_{t}
$$

and the conditional—on the current shock—expected growth rate, $\bar{\gamma}\left(s_{t} ; \theta, \rho\right)$, satisfies

$$
E_{t}\left\{y_{t+1} / y_{t}\right\} \equiv \bar{\gamma}\left(s_{t} ; \theta, \rho\right)=\varphi\left(s_{t} ; \theta, \rho\right)\left(1-\rho+\rho s_{t}\right)\left(s_{t} A+1-\delta\right) / s_{t}
$$

First, we want to describe how changes in the riskiness of the innovation, that is, $\theta$, affect the stochastic process for growth rates. To this end, we first describe how $\varphi\left(s_{t} ; \theta, \rho\right)$, the fraction of broad income saved, depends on $s$ and $\theta$. Formally, we show the following proposition.

Proposition 2: Assume that (2.4) holds. Then the optimal capital accumulation rule is of the form $k_{t+1}=\varphi\left(s_{t}, \theta\right)\left(s_{t} A+1-\delta\right) k_{t}$. Moreover, the function $\varphi(s, \theta)$ is

(i) increasing and concave in $s$ and decreasing in $\theta$, if $0<\sigma<1$,

(ii) constant and equal to $\beta$ if $\sigma=1$, 
(iii) decreasing and convex in $s$ and increasing in $\theta$, if $\sigma>1$.

Proof: See Appendix A.

Note that the conditional growth rate, $\bar{\gamma}\left(\mathrm{s}_{t} ; \theta, \rho\right)$, depends on $\theta$ only through $\varphi\left(s_{t} ; \theta, \rho\right)$. Thus, Proposition 2 shows that in the case of small risk aversion $(0<\sigma<1)$, increases in risk decrease the conditionally expected growth rate. If the utility function has more curvature than the $\log (\sigma>1)$, risk increases - as measured by $\theta$-result in conditional growth rate increases. Thus, for conditional growth rates, the role of curvature identified in the i.i.d. case remains unchanged.

What are the effects of changes in risk on expected or average growth rates? The expected growth rate is given by

$$
\bar{\gamma}(\theta, \rho)=\int s \varphi(s ; \theta, \rho)(1-\rho+\rho s)(s A+1-\delta) / s \lambda_{\theta}(d s)
$$

where $\lambda_{\theta}$ is the invariant measure of the process. ${ }^{14}$ It follows from our model that $\theta^{\prime}>\theta$ implies that $\lambda_{\theta}$ is dominated (in the second-order stochastic dominance sense) by $\lambda_{\theta^{\prime}}$.

Thus, if the integrand is convex, an increase in $\theta$ will increase $\bar{\gamma}(\theta, \rho)$, while if it is concave, the converse is true. We can now give a partial answer to the question of the effects of increased risk on average growth rates. In the case of high risk aversion $(\sigma>1)$, the function $\varphi(s ; \theta, \rho)$ is decreasing and convex, while the function $\left(1-\rho^{+} \rho s\right)(s A+1-\delta) / s$ is always convex. A sufficient condition for $\varphi(s ; \theta, \rho)\left(1-\rho^{+} \rho s\right)(s A+1-\delta) / s$ to be convex is that $\left(1-\rho^{+} \rho s\right)(s A+1-\delta) / s$ be decreasing for all values of $s$. This is equivalent to a restriction on the size of $\rho$. It is easy to check that since $s$ has support

${ }^{14}$ In this case, the invariant distribution is the distribution of the random variable $\sum_{j=0}^{\infty} \rho^{j} \varepsilon_{t-j}$, which inherits the changes in risk from the variables $\varepsilon_{t}$. 
in $[0,2]$, a sufficient condition for the expected growth rate to be a convex function of $s$ is that $\rho \leq(1-\delta) / 2(A+1-\delta)$. Thus, for moderate serial correlation, increases in riskiness, as measured by $\theta$, increase the expected growth rate. Of course, this condition is only sufficient, and in our numerical results we find that increases in the riskiness of the innovation process increase average growth rates (if $\sigma>1$ ), even for high values of the serial correlation coefficient, $\rho$.

When the utility function is logarithmic, $\varphi(s ; \theta, \rho)$ is constant and the growth rate is a strictly convex function of $s$ if $\delta<1$ and a linear function if $\delta=1$. This shows the role played by the depreciation rate. If it is small, which is the more realistic case, increases in the riskiness of the innovations increase average growth rates. Since this result is independent of the magnitude of the correlation coefficient, $\rho$, it applies to the i.i.d. case as well. Thus, the independence of the growth rate from the riskiness of the innovations that we found in Proposition 1 cannot, in general, surviveeven in an approximate sense-whenever depreciation rates are small. ${ }^{15}$

We summarize this discussion in the following proposition:

Proposition 3: Assume that the conditions of Proposition 2 hold. Then the expected—using the invariant measure — growth rate, $\bar{\gamma}(\theta, \rho)$, is strictly increasing in $\theta$ if

(i) $\sigma=1$, and $0<\delta<1$

(ii) $\sigma>1$, and $\rho \leq(1-\delta) / 2(A+1-\delta)$.

The case of small risk aversion, $0<\sigma<1$, is more complicated because the function $\varphi(s ; \theta$, $\rho)(1-\rho+\rho s)(s A+1-\delta) / s$ is the product of a concave and a convex function. Theory does not provide

\footnotetext{
${ }^{15}$ A continuity argument shows that if $\delta<1$, the average growth rate increases with uncertainty, even when $\sigma$ is less than (but close to) one.
} 
a prediction. Although we explored only small deviations from the log specification, we found that in all our numerical exercises, increases in $\theta$ increase expected growth rates even in this case.

We next explore the effects of changes in the serial correlation coefficient, $\rho$. It turns out that in our highly nonlinear model, it is not possible to give general results. To make some progress, we look at approximations (see Appendix B for derivations). Our basic finding is that if the fraction of broad income saved is not very sensitive to small shocks, the expected growth rate, $\bar{\gamma}(\theta$, $\rho)$, does not vary too much with $\rho$.

If preferences are logarithmic, the standard deviation of the growth rate, $\sigma_{\gamma}$, can be approximated by

$$
\sigma_{\gamma}=\left[2 v /(1+\rho)+(1-v)^{2} /\left(1-\rho^{2}\right)\right]^{1 / 2} \sigma_{\varepsilon}
$$

where $v \equiv(1-\delta) /(A+1-\delta)$ is a number close to 0.9 for reasonable specifications. It follows that increases in $\rho$, holding $\sigma_{s}$ constant, decrease $\sigma_{\gamma}$ and increases in $\sigma_{\varepsilon}$, holding $\sigma_{s}$ constant, increase $\sigma_{\gamma}$.

To study the autocorrelation of growth rates, we approximate (2.6) around $s_{t}=s_{t+1}=1$ to get

$$
\rho_{\gamma} \approx \rho+(\varsigma-v) /\left[1+(\varsigma-v)^{2}+\rho(\varsigma-v)\right]
$$

where $\varsigma \equiv \varphi_{s}(1 ; \theta, \rho) /\left.\varphi(s ; \theta, \rho)\right|_{s=1}$ is the elasticity of the (broad) savings rate with respect to the shock and $v$ is as before.

First, this expression shows that unless shocks are positively serially correlated, $\rho_{\gamma}$ is negative. To see this, take the limit as $\rho$ goes to zero and observe that, in that case, $\varphi\left(s_{t} ; \theta, \rho\right)$ is independent of $s_{t}$ and, hence, $\varsigma=0$. Thus, in the i.i.d. case,

$$
\rho_{\gamma} \approx-v /\left(1+v^{2}\right) \approx-0.5
$$

given that $v$ is close to one. ${ }^{16}$

\footnotetext{
${ }^{16}$ Note that this result does not coincide with our findings in the previous model with i.i.d. shocks due to the difference in depreciation. In that model, $\delta=1$, which implies, in our case, that $v=0$ and $\rho_{\gamma}=0$, as shown above.
} 
In order to generate growth rates more persistent than the fundamental shocks $\left(\rho_{\gamma}>\rho\right)$, it is necessary (but not sufficient) that $(\varsigma-v)>0$. For this to be the case, investment must respond positively to the shock; that is, $\varphi_{s}(1 ; \theta, \rho)>0$. From Proposition 2 , it follows that this can happen only if $0<\sigma<1$. It also follows that high depreciation rates (this corresponds to low $v$ ) help the model get persistence. In summary, for realistic parameter values, $\rho \approx 0$, then $\rho_{\gamma} \approx-1 / 2$. For $\rho_{\gamma}>\rho$, it is necessary that $\varphi_{s}>0$, and this requires that $\sigma<1$. If $\sigma>1$, then $\rho_{\gamma}<\rho$. Higher depreciation rates increase $\rho_{\gamma}$.

It is difficult to compare our results on the characterization of the effects of fundamental uncertainty on the distribution of growth rates with those in the real business cycle literature. The major problem is that in the real business cycle world, the curvature of the utility function plays a minor role, while in models of endogenous growth, it turns out to be a major determinant of the distribution of growth rates. The intuition for this is simple: differences in curvature play no role in the determination of the steady state of a standard Cass-Koopmans model, while the endogenous growth analog — a balanced growth path—depends crucially on the degree of intertemporal substitution. This different role played by the curvature parameter in the nonstochastic version remains in stochastic analyses, with the added impact that it balances income and substitution effects in determining the impact of additional risk on consumption and savings. In recent work, Fatas (2000) documents—-for a sample of 15 countries—a positive relationship between average growth and the serial correlation of growth rates. In his model, he assumes that differences in mean growth are due to differences in technology. Our results in this section complement Fatas' in that they describe how differences in the stochastic processes for fundamental uncertainty affect both mean growth rates and their second moments. 
Our findings in this section indicate that the relationship between fundamental shocks and the distribution of growth rates is highly nonlinear. We find that for curvature levels greater than or equal to the log—low intertemporal elasticity of substitution—increases in risk increase both investment over (properly defined) output ratios and average growth rates. Our results for curvature less than the log indicate that investment as a fraction of (properly defined) output decreases with risk. However, the implications for expected growth rates are less clear since only in the extreme case of i.i.d. shocks and full depreciation does the model give a robust prediction. Here, decreases in average growth follow from increases in risk.

The other two theoretical predictions of interest are, first, the differential impact of serial correlation and variability of innovations on the second moments of the growth rates and, second, the possibility of serially correlated growth rates if fundamental shocks are also serially correlated.

\section{Computing Equilibria of Linear Endogenous Growth Models}

The actual solutions to general versions of the models described in the preceding sections does cause some problems. The natural choice of the state is the vector $\left(k_{t}, h_{t}, s_{t}\right)$. The difficulty is that both $k_{t}$ and $h_{t}$ are converging to infinity (at least for versions of the model that exhibit growth on average). This renders numerical methods useless: they simply do not apply to this case. Despite this, the forms of the value and policy functions have relatively simple characterizations under some additional assumptions about the forms of the utility and production functions. The key property we will exploit is that for general versions of the models of the type described in (P.1) to have a balanced growth path, both preferences and technology must be restricted in a specific way (see King, Plosser, and Rebelo 1988 and Alvarez and Stokey 1998). Specifically, utility functions must be of the form 


$$
u(c, \ell)=\left\{\begin{array}{l}
v(\ell) c^{1-\sigma} /(1-\sigma) \quad \text { with } \sigma \neq 1, \text { but } \sigma>0 \\
\log (c)+v(\ell)
\end{array}\right.
$$

In our discussion, we will concentrate on the nonseparable case. The same arguments apply to the separable case.

On the technology side, we consider technology sets given by

$$
\begin{aligned}
& c_{t}+x_{z t}+x_{h t}+x_{k t} \leq F\left(k_{t}, z_{t}, s_{t}\right) \\
& z_{t} \leq M\left(n_{z t}, h_{t}, x_{z t}\right) \\
& k_{t+1} \leq\left(1-\delta_{k}\right) k_{t}+x_{k t} \\
& h_{t+1} \leq\left(1-\delta_{h}\right) h_{t}+G\left(n_{h t}, h_{t}, x_{h t}\right) \\
& \ell_{t}+n_{h t}+n_{z t} \leq 1
\end{aligned}
$$

$h_{0}$ and $k_{0}$ given. Here $\left\{s_{t}\right\}$ is a stochastic process which we assume is Markov with a transition probability function of $P(s, A)$ and in which $c_{t}$ is consumption, $x_{k t}$ is investment in physical capital, $k_{t}$ is the stock of physical capital, $x_{h t}$ is investment in human capital, $h_{t}$ is the stock of human capital, $z_{t}$ is effective labor, $n_{z t}$ is hours spent working in the market, $n_{h t}$ is hours spent augmenting human capital, and $\ell_{t}$ is leisure. The terms $\delta_{k}$ and $\delta_{h}$ are the depreciation rates on physical and human capital, respectively.

Thus, this is a fairly standard endogenous growth model in which effective labor is made up of a combination of hours and human capital which is supplied to the market. For specific choices of functional forms, many models in the literature are special cases of this formulation. For example, if $M=n_{z} h$ and $G=G_{0} h n_{h}$, the model corresponds to Lucas (1988) in the absence of externalities. If $M=n_{z} h$ and $G=x_{h}$, this corresponds to the two-capital-goods version discussed in Jones, Manuelli, and Rossi (1993). Finally, note that the standard one-sector growth model with exogenous technological change is also a special case (but the $s_{t}$ itself is not Markov in that case: $G \equiv 0, M(\bullet)=n_{z}$ ). 
Given the convexity of technologies and preferences, if markets are complete—as we assume-the equilibrium allocation can be found by solving a planner's problem of this form.

It can be shown that the essential property is that the technology set is linearly homogeneous in reproducible factors. This corresponds to the following restrictions:

(i) $\quad F$ is concave and homogeneous of degree one in $(k, z)$.

(ii) $M$ is concave and homogeneous of degree one in $\left(h, x_{z}\right)$.

(iii) $G$ is concave and homogeneous of degree one in $\left(h, x_{h}\right)$.

These restrictions effectively imply that the choice set in this more general version of (P.1) is linearly homogeneous in the initial stocks and that preferences are homothetic, holding fixed the nonreproducible choice variables, $n$ in our application. This implies that knowledge of the current shock and the current human capital/physical capital ratio (the two relevant pseudo state variables) is sufficient to determine the optimal choices of employment and the next period's human capital/physical capital ratio.

Let $\left\{e_{t}\right\}$ be the entire state/date contingent plan for the reproducible factors. The plan $\left\{e_{t}\right.$ $\left.n_{t}\right\}$ is feasible from initial state $e_{0}=\left(h_{0}, k_{0}\right)$, for a given $s_{0}$, if and only if $\left\{\lambda e_{t} n_{t}\right\}$ is feasible from the initial state $\lambda e_{0}=\left(\lambda h_{0}, \lambda k_{0}\right)(\lambda>0)$. Moreover, the utility (that is, the entire expected discounted sum) realized from $\left\{\lambda e_{t}, n_{t}\right\}$ is $\lambda^{1-\sigma}$ times the utility of $\left\{e_{t}, n_{t}\right\}$. Formally, consider the maximization problem,

(P.2) $\max U(e, n)$

subject to

$$
(e, n) \in \Gamma\left(h_{0}, k_{0}, s_{0}\right)
$$

where, as noted, $(e, n)$ is interpreted as the entire state/date contingent path of the endogenous variables and vector of labor supplies and $U$ is the resulting expected discounted sum of utilities. Let 
$V\left(h_{0}, k_{0}, s_{0}\right)$ denote the maximized value in this problem (assuming that it exists), and let $\left(e^{*}\left(h_{0}, k_{0}\right.\right.$, $\left.\left.s_{0}\right), n^{*}\left(h_{0}, k_{0}, s_{0}\right)\right)$ denote the optimal plan. Then we have the following proposition.

Proposition 4: Assume that the utility function in (P.2) is homogeneous of degree $1-\sigma$ in $e$ (holding $n$ fixed), that the feasible set, $\Gamma$, is linearly homogeneous in $(h, k)$ (holding $n$ and $s$ fixed), and that a solution exists for all $(h, k, s)$. Then the value function, $V$, for the problem (P.2) satisfies $V(\lambda k$, $\lambda h, s)=\lambda^{(1-\sigma)} V(k, h, s)$, for all $\lambda>0$. Moreover, the optimal plans are homogeneous of degree one in $z$ and degree zero in $n:\left(e^{*}(\lambda k, \lambda h, s), n^{*}(\lambda k, \lambda h, s)\right)=\left(\lambda e^{*}(h, k, s), n^{*}(h, k, s)\right)$.

\section{Proof: See Appendix A.}

From the point of view of a numerical approximation of problems like (P.2), this result implies that it is possible to estimate the optimal decision rules for $c / k, x_{j} / k, j=h, k, z$ as functions of the bounded—within a reasonably large set—variable $h / k$ and then calculate

$$
\begin{aligned}
& k^{\prime}=\left(1-\delta_{k}\right) k+k\left(x_{k} / k\right) \\
& h^{\prime}=\left(1-\delta_{h}\right) h+h G\left(x_{h} / k, h / k, n_{h}\right)
\end{aligned}
$$

to determine $h^{\prime} / k^{\prime}$. Thus, in this case, the Euler equations corresponding to (P.2) are solved by functions that depend only on the stationary variables $h / k$ and $s$.

Proposition 4 applies to any planning problem that has the required linearity and homogeneity properties. These include models with multiple sectors, preferences that depend on the state (for example, human capital determines effective leisure), and so on. A separate but related problem is under what conditions equilibrium allocations can be represented as solutions to planners' problems of the type described in (P.1). This class includes convex endogenous growth models with no 
external effects and the same class of models with proportional income taxes (see Jones and Manuelli 1999, among others).

Proposition 4 does not apply to planners' problems in which the technology displays increasing (for example, in Romer 1986) or decreasing (for example, in Brock and Mirman 1972 and the real business cycle application) returns to scale in reproducible factors or to problems that have distortions with no planning representations (for example, different tax rates on capital and labor income).

\section{Quantitative Effects of Uncertainty}

In this section, we rely on numerical methods to analyze the quantitative effects of variability in fundamentals on the distribution of growth rates.

\subsection{Model Specification and Calibration}

We study a special case of the model in Section 3. Specifically, we assume that $u(c, 1-n)=$ $\left[c(1-n)^{\psi}\right]^{1-\sigma} /(1-\sigma), F(k, z, s)=s A k^{\alpha} z^{1-\alpha}, G\left(n_{h t}, h_{t}, x_{h t}\right)=x_{h t}, M(n, h)=n h, s_{t+1}=1-\rho+\rho s_{t}+\varepsilon_{t+1}$, with $\varepsilon_{t}$ i.i.d., and $\varepsilon_{t} \sim U[-\zeta, \zeta]$.

The specification is standard. Our assumption that only $x_{h t}$ enters into the production of new human capital (which is, of course, produced using labor and both physical and human capital through the technology $F$ ) amounts to an aggregation assumption, namely, that the technology used to produce human capital as a function of capital and effective labor is identical to that in the final goods sector. Finally, we specify that $\delta_{k}=\delta_{h}$. This assumption greatly simplifies the solution because it implies a constant human capital/physical capital ratio (for details, see Appendix B).

To calibrate the model, we assume that $\alpha$, capital's share, is given by 0.36 , and we hold $\beta$ fixed at 0.95 . We assume that the common depreciation rate of human and physical capital is given 
by $\delta=0.075$, and in each case, we calibrate the model so that the labor supply in the nonstochastic steady state is given by $n=0.17$ (see Jones, Manuelli, and Rossi 1993). ${ }^{17}$ These restrictions still leave one degree of freedom in the selection of the parameters of preferences and technology. One way to solve this indeterminacy is to choose $\sigma$ (the coefficient of risk aversion) and the calibrated growth rate, $\gamma$. In order to do this consistently, we must simultaneously adjust $A$ (the average technology parameter) and $\psi$ (a curvature parameter in the utility function) to keep $n$ at 0.17 . We choose as our base case values of $\sigma=1.5$ and $\gamma=1.02$, or 2 percent growth per year.

To determine the stochastic process for the fundamental uncertainty, we must specify $\rho$ and $\zeta$. For our base case, we choose $\rho=0.9$ and $\zeta=0.08$. Unfortunately, there is no counterpart of $s_{t}$ in the data. However, different $\left\{s_{t}\right\}$ processes imply different stochastic processes for the growth rate. We use the average (across countries) standard deviation of the growth rate and its first-order serial correlation as the moments to match. In the Summers and Heston $(1991,1993)$ data set, the average (across countries) standard deviation of the per capita growth rate is 0.0601 and its serial correlation is 0.1256 . For our base case, the stochastic process given by $\rho=0.9$ and $\zeta=0.08$ comes close to replicating these values for the endogenously determined process for the growth rates. ${ }^{18}$

Even though our base case parameters are motivated by the desire to match observations, the principal aim of the paper is to understand how variability in fundamentals affects the distribution of growth rates more generally. Thus, we study alternative parameter values to better understand the effects of volatility on growth. First, our theoretical results indicate that some parameters-for

\footnotetext{
${ }^{17}$ In earlier versions of the paper, we also tried calibrations so that the nonstochastic steady-state labor supply was $n=0.3$. This had only minor effects; hence, the results are not included here.

${ }^{18}$ For our cross country data, we use the Penn World Tables 5.6. See Summers and Heston $(1991,1993)$.
} 
example, $\sigma, \rho$, and the standard deviation of the innovation $\sigma_{\varepsilon}$-are important determinants of the transmission mechanism of exogenous shocks. Second, the available evidence on growth rates from the Summers and Heston data set shows a large variation in average values across countries: even though the average across all countries is 1.98 percent, the first quartile is given by an average growth rate of 0.91 percent, while the third quartile is given by a rate of 3.23 percent. Since we want to explore the possibility that the heterogeneity in average growth is due to differences in the country-specific $\left\{s_{t}\right\}$ processes, we also consider changes in $\rho$ and $\sigma_{\varepsilon}$.

We study the following variations to our base case:

(a) We varied $\sigma$ from 0.9 to 3.0 .

(b) We varied the calibrated growth rate from 0 percent to 4 percent per year.

(c) We varied $\rho$ from 0.7 to 0.915 .

(d) We varied the standard deviation of the innovation from 0.035 to $0.081 .{ }^{19}$

Except in those exercises corresponding to the change in the nonstochastic mean (that is, point (b) above), every time a parameter is changed, the model is recalibrated to match the same moments as in the base case.

To solve the model, we compute the optimal decision rules after we discretize the state space. We then draw a realization of $\left\{s_{t}\right\}$ of size 5000 and compute the moments using this realization. In those cases in which the stochastic process $\left\{s_{t}\right\}$ is not changed, we have used the same realization to facilitate comparisons.

\footnotetext{
${ }^{19}$ It is easy to check that $\sigma_{\varepsilon}=(\zeta / 3)^{1 / 2}$. Thus, in terms of $\zeta$, we tried values from 0.06 to 0.14 .
} 


\subsection{Uncertainty, Risk Aversion, and Growth Rates}

In this section, we study how changes in the curvature of the utility function, $\sigma$, affect the distribution of growth rates. The basic results, for several specifications, are presented in Table 1. We report the values of $E(\gamma)$, the average growth rate in the simulation; $\sigma_{\gamma}$, the standard deviation of the growth rate in the simulation; and $\rho_{\gamma}$, the first-order autocorrelation coefficient of the growth rate in the simulation. For reference, we also present, in the last three rows, comparable statistics from the Summers and Heston (denoted SH) data set. Thus, the SH mean of 1.98 percent means that the average across countries is 1.98 percent, while the middle 50 percent of countries had average growth rates between 0.91 percent and 3.23 percent. Similarly, the average across countries of the standard deviation of the growth rate is 0.06 , while the middle 50 percent of countries had standard deviations between 0.041 and 0.077 . In this table, we hold $\rho$ and $\zeta$ fixed at their base case values of 0.9 and 0.08 , respectively, and adjust $\sigma$ from 0.9 to $3.0 .^{20}$

Since the nonstochastic version of all these specifications is calibrated to grow at 2 percent, any difference between the $E(\gamma)$ column and 2 percent is due to the increase in the standard deviation of the shock from 0 to 10 percent (as a percentage of the mean). The major findings are as follows:

(a) Our base case, corresponding to case 4 in Table 1, matches the standard deviation and first-order autocorrelation of the per capita growth rates fairly well. For this base case, the impact of increased uncertainty on mean growth is small and is approximately equal to onefifth of one percent per year. The largest impact of uncertainty occurs for preferences that are less concave than the log.

${ }^{20}$ Note that if $\sigma<1$, concavity of the utility function puts some restrictions on what $\psi$ can be. For each $\sigma$, we adjusted $A$ and $\psi$ to keep the growth rate for the nonstochastic version of the model fixed at 2 percent and the labor supply equal to 0.17 . Thus, we could equally well index the cases by either $A$ or $\psi$. 
(b) As suggested by Proposition 3, the average growth rate in the simulations exceeds the calibrated value of 2 percent for each value of $\sigma$.

(c) As expected, increases in the curvature of the utility function, $\sigma$, result in decreases in the standard deviation of growth rates. Thus, for coefficients of relative risk aversion exceeding one, we find that increases in risk aversion increase mean growth and decrease its variability. (d) For all these specifications, the model's prediction of the autocorrelation coefficient of growth rates is small, and often negative. It is clear that the growth rate of output-unlike the growth rate of capital—does not inherit the serial correlation properties of the driving shock.

(e) As expected from the theoretical results, the smaller the curvature of the utility function, the higher the autocorrelation coefficient. More curvature makes investment respond negatively to the current shock, and this, in turn, implies that the growth rate is more negatively serially correlated. At the other end, if the source of differences across economies is the curvature parameter, our model predicts a positive relationship between mean growth and the autocorrelation of growth rates if $\sigma$ is less than one. This is consistent with Fatas' (2000) finding.

(f) The effect of a given amount of uncertainty on the expected growth rate varies with the curvature parameter $\sigma$, and it is not a monotone function of curvature. Figure 1 shows that the largest impact of uncertainty occurs for values just below log utility. Moreover, for $\sigma>1$, increases in risk aversion increase $E(\gamma)$. Overall, the relationship between $\sigma$ and $E(\gamma)$ has a U-shape. 


\begin{tabular}{||c|c|c|c|c|c|c|c||}
\hline \hline \multicolumn{7}{|c|}{ Table 1. The Effect of $\sigma$ on $E(\gamma)$, the average growth rate } \\
\hline Case & $\sigma$ & $\rho$ & $\zeta$ & $\sigma_{s}$ & $E(\gamma)$ & $\sigma_{\gamma}$ & $\rho_{\gamma}$ \\
\hline 1 & 0.9 & 0.9 & 0.07 & 0.093 & $2.49 \%$ & 0.099 & 0.031 \\
\hline 2 & 1.0 & 0.9 & 0.08 & 0.106 & $2.34 \%$ & 0.090 & 0.012 \\
\hline 3 & 1.5 & 0.9 & 0.08 & 0.106 & $2.17 \%$ & 0.064 & -0.002 \\
\hline 4 & 2.0 & 0.9 & 0.08 & 0.106 & $2.19 \%$ & 0.059 & -0.005 \\
\hline 5 & 2.5 & 0.9 & 0.08 & 0.106 & $2.23 \%$ & 0.056 & -0.007 \\
\hline 6 & 3.0 & 0.9 & 0.08 & 0.106 & $2.28 \%$ & 0.055 & -0.008 \\
\hline SH mean & - & - & - & & $1.98 \%$ & 0.060 & 0.123 \\
\hline SH Q1 & - & - & - & & $0.91 \%$ & 0.041 & -0.039 \\
\hline SH Q3 & - & - & - & & $3.23 \%$ & 0.077 & 0.307 \\
\hline \hline
\end{tabular}

Overall, we find qualitatively that uncertainty affects growth in the expected direction. Quantitatively, the results are more difficult to interpret. The changes in average growth due to uncertainty range from one-fifth to one-half of one percent per year. Although the observed differences in average growth rates across countries in the Summers-Heston data set are substantially larger, it is not clear what fraction of these differences could potentially be due to differences in volatility.

\subsection{The Nature of Uncertainty and Its Effects on the Distribution of Growth Rates}

For the linear stochastic Markov process $\left\{s_{t}\right\}$, the standard deviation is $\sigma_{s}=\sigma_{\varepsilon} /\left(1-\rho^{2}\right)^{1 / 2}$. This moment depends on the magnitude of the standard deviation of the innovation, $\sigma_{\varepsilon}$, and the autocor- 
relation coefficient, $\rho$. In this section, we study the effects of varying these two components, $\sigma_{\varepsilon}$ and $\rho$, on the distribution of growth rates.

Our first set of experiments studies changes in $\sigma_{\varepsilon}$ given a value of $\rho .^{21}$ In the context of the theory developed in Section 2, an increase in $\sigma_{\varepsilon}$ corresponds to an increase in risk, $\theta$. In Figure 2 , we present the $\sigma=1.5$ case for two values of $\rho, 0.9$ and 0.8 . In this figure, we plot the effect of changes in $\sigma_{s}$ due to changes in $\sigma_{\varepsilon}$ on the expected growth rate. ${ }^{22}$ Our major findings are as follows:

(a) As expected, increases in $\sigma_{\varepsilon}$ increase the expected per capita growth rate. The impact is not linear, with larger effects for high levels of uncertainty. At the high end, when the standard deviation of the shock is 13.5 percent, the average growth rate is 2.8 percent, an increase of 0.8 percent over the deterministic benchmark. ${ }^{23}$

(b) The effect of a given change in $\sigma_{s}$ can have substantially different impacts on mean growth rates depending on the serial correlation of the shock. For example, for the $\rho=0.9$ economy (the dashed line in Figure 2), an increase in $\sigma_{s}$ from 10 percent to 12 percent has a very small impact on mean growth rates, while the same increase for the $\rho=0.8$ economy results in a substantial change in mean growth rates close to one-half of one percent. Based on these examples, it seems that the higher the level of serial correlation, the smaller the impact of variability on average growth. ${ }^{24}$

${ }^{21}$ Increases in $\zeta$ correspond to increases in $\sigma_{\varepsilon}$.

${ }^{22}$ See Appendix C, Table C.1, for the basic data.

${ }^{23}$ This is a substantial impact, but it comes at a high cost: In this case, the model predicts the standard deviation of the per capita growth rate to be 0.12 , twice the average value from the Summers and Heston data set, although still in the support of the distribution of standard deviations.

${ }^{24}$ This exercise also shows why we were forced to stay away from the standard linear-quadratic approximations used in the real business cycle literature. In the case of a linear approximation to the Euler equations, the theoretically predicted impact of changes in $\sigma_{\varepsilon}$ on the decision rules is zero. It is because of our interest in this higher-order effect that we used a different numerical strategy. 
(c) Changes in $\sigma_{s}$ given by changes in $\sigma_{\varepsilon}$ have almost linear effects on the standard deviation of the growth rate and very small effects on the autocorrelation of growth rates (Table C.1 in Appendix C).

For our next set of experiments, we hold $\sigma_{\varepsilon}$ constant and change $\sigma_{s}$ through changes in the correlation coefficient of the driving shocks, $\rho$. The major findings (the basic data are in Tables C.1 and C.2 in Appendix C) are as follows:

(a) Increases in $\rho$ (holding $\sigma_{\varepsilon}$ constant) have small but negative effects on the average growth rate (Table C.2 in Appendix C contains the data).

(b) Increases in $\rho$ have a small but negative, as expected, impact on the standard deviation of growth rates.

(c) Increases in $\rho$ increase the serial correlation of the growth rate (see Table C.2 in Appendix C).

Is it possible that uncertainty has a different effect for high growth and low growth countries $?^{25}$ To explore this issue, we tried adjusting the nonstochastic steady-state growth rate to which we calibrate the model. For our base case, we tried several values from 0 percent to 4 percent. Our numerical results (see Table C.3 in Appendix C) show that the nonstochastic steady state has no impact on measured moments of the distribution of growth rates.

\subsection{Volatility and Cyclical Behavior}

Even though our primary interest in this paper is to begin the exploration of the effects of uncertainty on growth, our model delivers implications for cyclical variables. However, unlike developers of more standard real business cycle models, we are not free to detrend the data. Our theoretical model

\footnotetext{
${ }^{25}$ In the context of this paper, the differences in growth rates could be due to distortionary taxes or differences in technology or both.
} 
implies that the appropriate detrending procedure is to consider the ratio of each variable, except hours worked (our variable $n$ ), to output. Our model implies that hours worked is a stationary variable.

Before we confront our model's predictions with the data, we must match the notion of investment in human capital with observable quantities. In the model, the variable $x_{h}$ corresponds to investment and is conceptually different from consumption. What is the counterpart in the data? This question is difficult because it is not clear what human capital is. Most economists would probably agree that human capital includes education and training, but it is also likely to encompass other activities like health care and investments in mobility. Even for those items for which there is consensus (for example, education and training), there are no good measures. To say the least, training is poorly measured and, depending on its nature, may not even be part of measured output. In the case of education, and some forms of training, gross investments appear in consumption. ${ }^{26}$ Thus, in this paper, we assume that all of $x_{h}$ is part of measured output, and we experiment with two notions of consumption: the narrow view that consumption in the data corresponds to consumption in the model and the broad view that consumption in the data is the sum of consumption and investment in human capital, $c+x_{h \cdot}{ }^{27}$

In Table 2, we report the results for our base case and for various levels of curvature. There are four interesting features:

(a) Uncertainty has a small effect on the mean of the consumption/output ratio, both in its narrow version, $c / y$, and its broad version, $\left(c+x_{h}\right) / y$. However, the choice of narrow versus

\footnotetext{
${ }^{26}$ Of course, it is possible to net out educational expenditures, both private and public; however, other components like health care are much more difficult to allocate since not all expenditures probably qualify as investments in productive human capital.

${ }^{27}$ For an extended discussion of alternative strategies in terms of allocating $x_{h}$ in different ways, see Jones, Manuelli, Siu, and Stacchetti (1998).
} 
broad consumption has a substantial effect on the mean value of $c / y$, adding roughly 30 percentage points.

(b) The model has the very sharp implication that higher values of relative risk aversion result in decreases in the standard deviation of the consumption/output ratio using either measure. This decrease is dramatic. The standard deviation increases sixfold when moving from $\sigma=3.0$ to $\sigma=0.9$. For reference, the standard deviation of measured $c / y$ in the United States is $0.019 .^{28}$ If we want the model to match the standard deviation of the consumption/output ratio, the best estimate of $\sigma$ is slightly above one.

(c) The model implies that the amount of curvature in the utility function has sharp implications for the coefficient of variation of the number of hours worked. The basic data are in the last column of Table 2. As the coefficient of relative risk aversion moves from 3.0 to 0.9 , the predicted coefficient of variation of the number of hours worked increases by a factor of eight. For comparative purposes, the analogous value of the coefficient of variation of hours worked in the United States is $0.034 .^{29}$ Thus, in this case, the best value of $\sigma$ is something close to 2 .

(d) In the cases presented to this point, $n(s)$ is strictly increasing as a function of $s$. However, it is possible to modify the model and get a nonlinear $n(s)$ function. Our results (not pre-

${ }^{28}$ Let $\sigma\left(\left(c+x_{h}\right) / y\right)$ be the standard deviation of a broad measure of consumption that includes investment in human capital, $x_{h}$, as part of consumption, and let $\sigma(c / y)$ be the analog for a narrow notion of consumption. It follows that $\sigma\left(\left(c+x_{h}\right) / y\right)=\alpha \sigma(c / y)$. Thus, broad consumption is less variable than narrow consumption because the former includes $x_{h}$, which is an investment good, and, as such, its ratio to output increases in good times and decreases in bad times. The curvature in the utility function implies that the $c / y$ ratio decreases in good times and increases in bad times. Thus, roughly, $c / y$ and $x_{h} / y$ are negatively correlated. Hence, their sum exhibits lower variability than either of the components.

${ }^{29}$ For the U.S. data, we use the Burnside and Eichenbaum (1994) data. To calculate the coefficient of variation of hours worked, we did not detrend the per capita number of hours. 
sented here) suggest that cases in which the mean growth rate is small (say, less than 1.4 percent) and the serial correlation of the shock is large (exceeding 0.95) are consistent with an increasing response of hours worked to productivity shocks when the shock is small and a decreasing response when the shock is large. Whether that asymmetric response can account for puzzles like the productivity slowdown and the behavior of hours worked over the cycle we do not know at this point.

\begin{tabular}{||c|c|c|c|c|c|c|c|c||}
\hline \hline \multicolumn{8}{|c|}{ Table 2. Properties of Consumption and Labor Supply } \\
\hline Case & $\sigma$ & $\rho$ & $\zeta$ & $E(c / y)$ & $E\left(\left(c+x_{h}\right) / y\right)$ & $\sigma(c / y)$ & $\sigma\left(\left(c+x_{h}\right) / y\right)$ & $\sigma(n) / E(n)$ \\
\hline 1 & 0.9 & 0.9 & 0.07 & 0.37 & 0.77 & 0.089 & 0.032 & 0.186 \\
\hline 2 & 1.0 & 0.9 & 0.08 & 0.37 & 0.77 & 0.068 & 0.024 & 0.137 \\
\hline 3 & 1.5 & 0.9 & 0.08 & 0.41 & 0.79 & 0.027 & 0.010 & 0.054 \\
\hline 4 & 2.0 & 0.9 & 0.08 & 0.44 & 0.80 & 0.019 & 0.007 & 0.035 \\
\hline 5 & 2.5 & 0.9 & 0.08 & 0.47 & 0.81 & 0.016 & 0.006 & 0.027 \\
\hline 6 & 3.0 & 0.9 & 0.08 & 0.50 & 0.82 & 0.014 & 0.005 & 0.022 \\
\hline \hline
\end{tabular}

\section{Conclusion}

For the class of neoclassical models that we study, changes in the variability of fundamentals also result in changes in average growth rates. For levels of risk aversion at least as high as the log, eliminating cycles completely would result in lower growth rates. The size of this effect ranges from 0.2 percent per year to 0.5 percent per year, depending on the parameters of preferences. Of course, this only reinforces Lucas' conclusions that the payoff from eliminating cycles is not too large.

Theoretically, we show that increased uncertainty can decrease average growth. However, this requires parameter values that lie outside the usual range: high intertemporal substitution, no 
correlation of major shocks, and very short-lived capital.

We also identify, for reasonable parameter values, changes in the variability of the innovations to fundamental shocks as having a larger impact on average growth rates than changes in the serial correlation of the shocks.

From a quantitative point of view, there are two major findings. First, for reasonable values of exogenous uncertainty, variability in fundamentals is not large enough to be the only reason average growth rates differ so much across countries. In addition, differences in stochastic processes for the fundamental shocks do not give rise to a positive relationship between mean growth rates and their autocorrelation coefficients. Second, uncertainty in fundamentals has a large impact on the predicted standard deviation of cyclical variables (for example, the consumption/output ratio), and the size of the impact is sensitive to the degree of curvature of preferences.

Our finding that increased uncertainty increases average growth seems at odds with the empirical work of Ramey and Ramey (1995). However, since the shocks in our model can be interpreted as shocks to tax rates, our results imply that-holding average tax rates fixed-increases in the variance of tax rates increase average growth. Of course, if growth-inhibiting policies (on average) are associated with volatile policies, the model could deliver a negative correlation between volatility and average growth. However, in this case, it is not the high volatility that is causing growth to be low, but the high average tax rates. ${ }^{30}$

Our preliminary conclusion is that, even though there is a trade-off between fluctuations and growth, bringing stochastic elements to the class of endogenous growth models that we studied does

${ }^{30}$ Ramey and Ramey (1995) find that policy variability is associated with residual uncertainty. Our findings do not depend on the shock affecting all sectors. The model in Obstfeld (1994) can be used to show that for risk aversion levels greater than the log, there is an approximate positive relationship between variability and growth. This relationship is approximate because in the model, the relationship between variability of output and mean output is not a function, but a correspondence. 
not radically improve those models' ability to explain growth facts. However, the model delivers sharp implications about the effect of curvature in preferences on the variability of cyclical variables and, hence, can use data to pin down preference parameters. The version of the model that we studied is too simple to proceed with this program. One manifestation of this is the difficulty in matching growth and cyclical observations simultaneously. In ongoing work (see Jones, Manuelli, Siu, and Stacchetti 1998), we study versions of these models that allow for variable human capital/physical capital ratios and different specifications of the human capital augmentation technology. 


\section{Appendix A: Proofs}

Proof of Proposition 1: We first consider the case in which $\sigma \neq 1$. Consider the first model of Section 2. The first-order conditions are

(A.1.1) $\quad c_{t} v^{\prime}\left(n_{t}\right)=(\sigma-1)(1-\alpha) y_{t} v\left(n_{t}\right) / n_{t}$

(A.1.2) $\quad c_{t}^{-\sigma} v\left(n_{t}\right)=\beta \int_{S}\left[c_{t+1}^{-\sigma} v\left(n_{t+1}\right)\right]\left[A \alpha k_{t+1}^{\alpha-1} h_{t+1}^{1-\alpha} n_{t+1}^{1-\alpha}\left(1+\varepsilon_{t+1}\right)\right] \mu_{\theta}\left(d \varepsilon_{t+1}\right)$

(A.1.3) $\quad c_{t}^{-\sigma} v\left(n_{t}\right)=\beta \int_{S}\left[c_{t+1}^{-\sigma} v\left(n_{t+1}\right)\right]\left[A(1-\alpha) k_{t+1}^{\alpha} h_{t+1}^{-\alpha} n_{t+1}^{1-\alpha}\left(1+\varepsilon_{t+1}\right)\right] \mu_{\theta}\left(d \varepsilon_{t+1}\right)$

and the feasibility constraints at equality. In order to find the solution to the planner's problem, we first hypothesize that (A.1.2) and (A.1.3) are satisfied by having the terms in square brackets inside the integral operator equal in each state. Second, we conjecture that consumption is a constant fraction of income. Finally, we guess that the fraction of the time allocated to working is constant as well. These conjectures imply that the solution must satisfy

(A.1.4) $h_{t}=[(1-\alpha) / \alpha] k_{t}$

(A.1.5) $(1-\varphi) v^{\prime}(n)=(\sigma-1)(1-\alpha) v(n) / n_{t}$

(A.1.6) $\quad \varphi^{\sigma}=\beta\left(A^{*}\right)^{1-\sigma} n^{(1-\alpha)(1-\sigma)} \int_{S}\left(1+\varepsilon_{t+1}\right)^{1-\sigma} \mu_{\theta}\left(d \varepsilon_{t+1}\right)$

where $\varphi$ is the fraction of income, $y$, which is saved (of course, $1-\varphi$ is consumed), and $A^{*}$ is $A(1-\alpha)^{(1-\alpha)} \alpha^{\alpha}$. The solution to equations (A.1.5) and (A.1.6) can be used to construct an equilibrium by letting investment in physical capital, $x_{k}$ be given $\alpha \varphi y$, while $x_{h}$ is $(1-\alpha) \varphi y$. To simplify notation, let $D=\left[\beta\left(A^{*}\right)^{(1-\sigma)}\right]^{1 / \sigma}$, and let $\hat{s}=\int_{S}(1+\varepsilon)^{(1-\sigma)} \mu_{\theta}(d \varepsilon)$. Then (A.1.5) and (A.1.6) imply that the equilibrium values of $\varphi$ and $n$ solve

$$
\begin{aligned}
& \varphi=H(n) \equiv 1-\left[(\sigma-1)(1-\alpha) v(n) /\left(n v^{\prime}(n)\right)\right] \\
& \varphi=G(n) \equiv D \hat{s}^{1 / \sigma} n^{(1-\alpha)(1-\sigma) / \sigma}
\end{aligned}
$$

which correspond to equations (2.1) and (2.2) in the text. 
Note that the function $G(n)$ is upward sloping if $0<\sigma<1$ and downward sloping if $\sigma>1$. Moreover, increases in $\hat{s}$ increase $G(n)$. The properties of $H(n)$ depend on $v$. However, concavity of the utility function imposes some restrictions. The nature of these restrictions depends on $\sigma$. It is straightforward to verify that positive marginal utility of leisure and concavity imply that $v^{\prime}(n) /(1-\sigma)$ and $v^{\prime \prime}(n) /(1-\sigma)$ must both be negative. In addition, concavity requires that $(\sigma /(\sigma-1)) v^{\prime \prime}(n) v(n)-\left(v^{\prime}(n)\right)^{2}>0$. To ensure that these conditions hold for all values of $\sigma$, we will assume that $v^{\prime \prime}(n) v(n)-\left(v^{\prime}(n)\right)^{2}>0$. These restrictions imply that $H(n)$ is an increasing function of n. Finally, note that (C.1) simply states that $H(1)>G(1)$.

We first discuss existence and uniqueness for the two possible ranges of $\sigma$. Consider the case in which $\sigma>1$. It follows that

$$
\lim _{n \rightarrow 0} G(n)=\infty, G(1)=D \hat{s}^{1 / \sigma}, \text { and } G^{\prime}(n)<0
$$

and

$$
\lim _{n \rightarrow 0} H(n)<\infty, H(1)>G(1), \text { and } H^{\prime}(n)>0 .
$$

It follows that there is a unique intersection. An example is shown in Figure A.1.

Consider next the case in which $0<\sigma<1$. In this case, we have that

$$
\lim _{n \rightarrow 0} G(n)=0, G(1)=D \hat{s}^{1 / \sigma}, \text { and } G^{\prime}(n)>0
$$

and

$$
\lim _{n \rightarrow 0} H(n)<0, H(1)>G(1), \text { and } H^{\prime}(n)>0
$$

where the first inequality corresponds to (C.2). The problem here is that both $H(n)$ and $G(n)$ are upward sloping, and establishing uniqueness requires a separate argument. It is possible to show (details available from the authors) that if $n^{*}$ satisfies $G\left(n^{*}\right)=H\left(n^{*}\right)$, then $H^{\prime}\left(n^{*}\right)>G^{\prime}\left(n^{*}\right)$. Thus, the 
function $H$ can intersect the function $G$ only from below. This, of course, suffices for uniqueness. Possible $H(n)$ and $G(n)$ functions are displayed in Figure A.2.

In Figures A.1 and A.2, we use $G^{\prime}$ to denote the function $G$ corresponding to a higher value of $\hat{s}^{1 / \sigma}$. Thus, it follows that increases in $\hat{s}^{1 / \sigma}$ increase both the number of hours allocated to working (the utilization rate of human capital), $n$, and the fraction of income saved, $\varphi$. It is straightforward to calculate the growth rate of output. It is given by

$$
y_{t+1} / y_{t} \equiv \gamma_{t+1}=s_{t+1} A^{*} n^{1-\alpha} \varphi=s_{t+1} \gamma
$$

Thus, the average growth rate, $\gamma$, is simply $\mathrm{A}^{*} n^{1-\alpha} \varphi$. It follows that growth rates are increasing in $\hat{s}$. Let $\hat{s}(\theta)$ be given by $\hat{s}(\theta)=\int_{S}(1+\varepsilon)^{(1-\sigma)} \mu_{\theta}(d \varepsilon)$. Since the function $(1+\varepsilon)^{(1-\sigma)}$ is concave for $0<\sigma<1$ and convex for $\sigma>1$, it follows that $0<\sigma<1, \hat{s}(\theta)$ is increasing, and if $\sigma>$ $1, \hat{s}(\theta)$ is decreasing. This, in turn, implies that $[\varphi, n, \gamma]$ are decreasing in $\theta$ whenever $0<\sigma<1$ and increasing otherwise.

From $\gamma_{t+1}=\mathrm{s}_{t+1} \gamma$, it follows that $\sigma_{\gamma}=\gamma \sigma_{s}$, where $\sigma_{s}$ is the standard deviation of the shock, $s_{t}$. Thus, $\sigma_{\gamma} / \sigma_{s}=\gamma$, and our claims follow from the properties of $\gamma$.

Now consider the case in which $\sigma=1$. The first-order conditions are satisfied with $\varphi=\beta$ and $n$ as the unique solution to $n v^{\prime}(n)=(\alpha-1) /(1-\beta)$. It is clear that, in this case, the key elements of the equilibrium are independent of $\theta$.

Proof of Proposition 2: Here we present the proof for the case in which $\sigma>1$. The same type of arguments (with the signs suitably reversed) apply to the $0<\sigma<1$ case. The relevant first-order condition for the planner's problem is

$$
c_{t}^{-\sigma}=\beta \int c_{t+1}^{-\sigma}\left[A s_{t+1}+1-\delta\right] P_{\theta}\left(s_{t}, d s_{t+1}\right) .
$$

Under the guess $k_{t+1}=\varphi\left(s_{t}, \theta\right)\left(s_{t} A+1-\delta\right) k_{t}$, this first-order condition can be written as 


$$
[1-\varphi(s, \theta)]^{-\sigma}=\beta \int[1-\varphi(z, \theta)]^{-\sigma}[\varphi(s, \theta)]^{-\sigma}[A x+1-\delta]^{1-\sigma} P_{\theta}(s, d x)
$$

or

(A.2.1) $f(s, \theta)=1+\left(\beta \int\left[f(1-\rho+\rho s+\varepsilon, \theta)(A(1-\rho+\rho s)+1-\delta+A \varepsilon)^{1-\sigma / \sigma}\right]^{\sigma} \mu_{\theta}(d \varepsilon)\right)^{1 / \sigma}$

where $f(s, \theta)=[1-\varphi(s, \theta)]^{-1}$.

Let the right-hand side of (A.2.1) define the mapping $T_{\theta}$. We want to show that $T_{\theta}$ is a contraction mapping. To this end, we will show that $T_{\theta}$ maps a set, $\mathscr{T}$, of continuous, bounded, and convex functions into itself and that $T_{\theta}$ satisfies Blackwell's conditions for a contraction.

Let $\mathscr{F} \equiv\{f: S \rightarrow \Re$, such that $1 \leq f(s) \leq \bar{f}, f(s)$ decreasing, continuous, and convex $\}$. Let $\bar{f}$ be given by

$$
\bar{f}=\sup _{s} 1 /\left[1-\left(\beta \int\left(A\left(1-\rho^{+} \rho s\right)+1-\delta+A \varepsilon\right)^{1-\sigma} \mu_{\theta}(d \varepsilon)\right)^{1 / \sigma}\right]
$$

which is finite given our assumptions. It follows that if $f(s) \leq \bar{f}$, then $T_{\theta} f(s) \leq \bar{f}$. It follows that if $f(s)$ is decreasing and continuous, so is $T_{\theta} f(s)$. We next show that $T_{\theta}$ maps convex functions into convex functions. Let $s^{\lambda}=\lambda s^{1}+(1-\lambda) s^{2}$ for some $0<\lambda<1$. Let $f$ be convex and decreasing. Define

$$
\begin{aligned}
& m(\varepsilon) \equiv \lambda f\left(1-\rho+\rho s^{1}+\varepsilon\right)\left(A\left(1-\rho+\rho s^{1}\right)+1-\delta+A \varepsilon\right)^{1-\sigma / \sigma} \\
& g(\varepsilon) \equiv(1-\lambda) f\left(1-\rho^{+} \rho s^{2}+\varepsilon\right)\left(A\left(1-\rho^{+} \rho s^{2}\right)+1-\delta+A \varepsilon\right)^{1-\sigma / \sigma}
\end{aligned}
$$

For any $\varepsilon$, the function $f(1-\rho+\rho s+\varepsilon)(A(1-\rho+\rho s)+1-\delta+A \varepsilon)^{1-\sigma / \sigma}$ is a convex function of $s$. Thus,

$$
\left[f\left(1-\rho^{+} \rho s^{\lambda}+\varepsilon\right)\left(A\left(1-\rho^{+} \rho s^{\lambda}\right)+1-\delta+A \varepsilon\right)^{1-\sigma / \sigma}\right]^{\sigma} \leq[m(\varepsilon)+g(\varepsilon)]^{\sigma}
$$

and

$$
\left(\beta \int\left[f\left(1-\rho+\rho s^{\lambda}+\varepsilon\right)\left(A\left(1-\rho+\rho s^{\lambda}\right)+1-\delta+A \varepsilon\right)^{1-\sigma / \sigma}\right]^{\sigma} \mu_{\theta}(d \varepsilon)\right)^{1 / \sigma} \leq\left(\beta \int[m(\varepsilon)+g(\varepsilon)]^{\sigma} \mu_{\theta}(d \varepsilon)\right)^{1 / \sigma}
$$

From Minkowski's inequality (see Rudin 1974, Theorem 3.5, p. 65), it follows that

$$
\left(\beta \int[m(\varepsilon)+g(\varepsilon)]^{\sigma} \mu_{\theta}(d \varepsilon)\right)^{1 / \sigma} \leq\left(\beta \int m(\varepsilon)^{\sigma} \mu_{\theta}(d \varepsilon)\right)^{1 / \sigma}+\left(\beta \int g(\varepsilon)^{\sigma} \mu_{\theta}(d \varepsilon)\right)^{1 / \sigma}
$$


Using (A.2.2) and (A.2.3) and adding one to both sides, we get

$$
\begin{aligned}
& 1+\left(\beta \int\left[f\left(1-\rho+\rho s^{\lambda}+\varepsilon\right)\left(A\left(1-\rho+\rho s^{\lambda}\right)+1-\delta+A \varepsilon\right)^{1-\sigma / \sigma}\right]^{\sigma} \mu_{\theta}(d \varepsilon)\right)^{1 / \sigma} \leq \lambda\left[1+\left(\beta \int \left[f\left(1-\rho+\rho s^{1}+\varepsilon\right)\right.\right.\right. \\
& \left.\left.\left.\left(A\left(1-\rho+\rho s^{1}\right)+1-\delta+A \varepsilon\right)^{1-\sigma / \sigma}\right]^{\sigma} \mu_{\theta}(d \varepsilon)\right)^{1 / \sigma}\right]+(1-\lambda)\left[1+\left(\beta \int \left[f ( 1 - \rho + \rho s ^ { 2 } + \varepsilon ) \left(A\left(1-\rho+\rho s^{2}\right)\right.\right.\right.\right. \\
& +1-\delta+A \varepsilon) 1-\sigma / \sigma] \sigma \mu \theta(d \varepsilon)) 1 / \sigma]
\end{aligned}
$$

or

(A.2.4) $\quad T_{\theta} f\left(s^{\lambda}\right) \leq \lambda T_{\theta} f\left(s^{1}\right)+(1-\lambda) T_{\theta} f\left(s^{2}\right)$.

Thus, $T_{\theta}$ preserves convexity. To show that $T_{\theta}$ is a contraction mapping, it suffices to show that it satisfies the conditions of Blackwell's theorem (see Stokey and Lucas 1989). These conditions are

(A.i) (monotonicity) $f \geq g \rightarrow T_{\theta} f \geq T_{\theta} g$

(A.ii) (discounting) there exists a $v$ satisfying $0<v<1$, such that for all $a \in \Re_{+}$,

$$
T_{\theta}(f+a) \leq T_{\theta} f+v a
$$

It is immediate that (A.i) is satisfied. To prove (A.ii), use Minkowski's inequality to show that

$$
\begin{aligned}
& 1+\left(\beta \int\left[f\left(1-\rho^{+} \rho s^{+} \varepsilon\right)\left(A\left(1-\rho^{+} \rho s\right)+1-\delta+A \varepsilon\right)^{1-\sigma / \sigma}+a\left(A\left(1-\rho^{+} \rho s\right)+1-\delta+A \varepsilon\right)^{1-\sigma / \sigma}\right]^{\sigma} \mu_{\theta}(d \varepsilon)\right)^{1 / \sigma} \\
& \leq 1+\left(\beta \int\left[f\left(1-\rho^{+} \rho s^{+} \varepsilon\right)\left(A\left(1-\rho^{+} \rho s\right)+1-\delta+A \varepsilon\right)^{1-\sigma / \sigma}\right]^{\sigma} \mu_{\theta}(d \varepsilon)\right)^{1 / \sigma} \\
& +\left(\beta \int\left[a\left(A\left(1-\rho^{+} \rho s^{2}\right)+1-\delta+A \varepsilon\right)^{1-\sigma / \sigma}\right]^{\sigma} \mu_{\theta}(d \varepsilon)\right)^{1 / \sigma}
\end{aligned}
$$

or, equivalently,

$$
T_{\theta}(f+a) \leq T_{\theta} f+a\left(\beta \int\left[\left(A\left(1-\rho+\rho s^{2}\right)+1-\delta+A \varepsilon\right)^{1-\sigma / \sigma}\right]^{\sigma} \mu_{\theta}(d \varepsilon)\right)^{1 / \sigma} \leq T_{\theta} f+a \kappa^{1 / \sigma}
$$

where $\kappa$ is defined in (2.4) and was assumed to be less than one.

It then follows that $T_{\theta}$ is a contraction mapping and that it has a unique fixed point $f(s, \theta)$. Moreover, this fixed point is decreasing and convex in $s$. 
We now show that if $\theta^{\prime}>\theta$, then $f\left(s, \theta^{\prime}\right)>f(s, \theta)$. To see this, note that

$$
T_{\theta^{\prime}} f(s, \theta) \leq T_{\theta} f(s, \theta)=f(s, \theta)
$$

and

$$
T_{\theta^{\prime}}^{2} f(s, \theta) \geq T_{\theta^{\prime}} T_{\theta} f(s, \theta) \geq T_{\theta}^{2} f(s, \theta)=f(s, \theta)
$$

where the first inequality follows from (A.i) and the second follows because $T_{\theta} f(\bullet, \theta)$ is a convex function. Similar arguments show that

$$
f\left(s, \theta^{\prime}\right)=\lim _{n \rightarrow \infty} T_{\theta^{\prime}}^{n} f(\cdot, \theta) \geq f(s, \theta) .
$$

It is easy to check that Minkowski's inequality cannot hold as an equality (see Rudin 1974) and, hence, that the above inequality is strict.

Since $\varphi(s, \theta)=1-f(s, \theta)^{-1}$, it follows that $\varphi(s, \theta)$ inherits the properties of $f(s, \theta)$; namely, it is decreasing and convex.

Proof of Proposition 4: Fix an arbitrary initial state $(h, k, s)$, and let $\left(z^{*}(h, k, s), n^{*}(h, k, s)\right)$ denote the solution to (P.2) from this state. Now consider the same problem when the initial state is $(\lambda k$, $\lambda h, s)$. It follows immediately from the linear homogeneity of $\Gamma$ that $\left(\lambda z^{*}(h, k, s), n^{*}(h, k, s)\right)$ is feasible for the problem with initial state $(\lambda k, \lambda h, s)$. Contrary to the conclusion of the proposition, assume that $\left(\lambda z^{*}(h, k, s), n^{*}(h, k, s)\right)$ is not optimal. Then take some alternative plan, $(z, n)$, that is feasible and gives higher utility:

$$
\text { (A.4.1) } U(z, n)>U\left(\lambda z^{*}(h, k, s), n^{*}(h, k, s)\right) \text {. }
$$

Since $(z, n)$ is feasible given initial state $(\lambda k, \lambda h, s)$, it follows from the linear homogeneity of $\Gamma$ that $(z / \lambda, n)$ is feasible when the initial state is $(\lambda k / \lambda, \lambda h / \lambda, s)=(h, k, s)$. Moreover, the utility of $(z / \lambda, n)$ is given by $U(z / \lambda, n)=U(z, n) / \lambda^{1-\sigma}$. Using this and (A.4.1), we have that 


$$
U(z / \lambda, n)=U(z, n) / \lambda^{1-\sigma}>U\left(\lambda z^{*}, n^{*) /} \lambda^{1-\sigma}=\lambda^{1-\sigma} U\left(z^{*}, n \lambda^{1-\sigma}=U\left(z^{*}, n^{*}\right) .\right.\right.
$$

That is, $(z / \lambda, n)$ is feasible when the initial state is $(h, k, s)$, and it gives higher utility than $\left(z^{*}, n^{*}\right)$, a contradiction.

That the value function is homogeneous of degree $1-\sigma$ in $z$ (holding $n$ fixed) follows immediately from the fact that the policy rules have the property that they do. 


\section{Appendix B: Derivations}

Approximations for the Second Moments of Growth Rates

First, we study the effects of changing $\rho$ on the mean growth rate. From the proof of Proposition 2, it follows that $\varphi(s ; \theta, \rho)$ solves

$$
[1-\varphi(s ; \theta, \rho)]^{-\sigma}=\left[1+\left(\beta \int[1-\varphi(1-\rho+\rho s+\varepsilon ; \theta, \rho)]^{-\sigma}[A(1-\rho+\rho s+\varepsilon)+1-\delta]^{1-\sigma} \mu_{\theta}(d \varepsilon)\right)^{1 / \sigma}\right]^{\sigma} .
$$

Differentiating with respect to $\rho$ and evaluating at $s=1$, and if we get

$$
\begin{aligned}
& \varphi_{\rho}(1 ; \theta, \rho) /(1-\varphi(1 ; \theta, \rho))=\left[1+\left(\beta \int[1-\varphi(1+\varepsilon ; \theta, \rho)]^{-\sigma}[A(1+\varepsilon)+1-\delta]^{1-\sigma} \mu_{\theta}(d \varepsilon)\right)^{-1 / \sigma}\right]^{-1} \\
& \left\{\int \varphi_{\rho}(1+\varepsilon ; \theta, \rho) /(1-\varphi(1+\varepsilon ; \theta, \rho)) \psi(d \varepsilon)\right\}
\end{aligned}
$$

where $\psi(d \varepsilon)$ is just $[1-\varphi(1+\varepsilon ; \theta, \rho)]^{-\sigma}[A(1+\varepsilon)+1-\delta]^{1-\sigma} \mu_{\theta}(d \varepsilon) / \int[1-\varphi(1+\varepsilon ; \theta, \rho)]^{-\sigma}[A(1+\varepsilon)+1-\delta]^{1-\sigma}$ $\left.\mu_{\theta}(d \varepsilon)\right)$, it follows that if the fraction saved does respond very strongly to a small shock, $\varphi_{\rho}(1+\varepsilon ; \theta$, $\rho) /\left(1-\varphi(1+\varepsilon ; \theta, \rho)\right.$ is approximately constant and, hence, $\varphi_{\rho}(1 ; \theta, \rho) /(1-\varphi(1 ; \theta, \rho)) \approx 0$.

To study the serial correlation properties of growth rates, we linearize the function that defines the growth rate. We can then approximate (2.6) around $s_{t}=s_{t+1}=1$ by

$$
\gamma\left(s_{t}, s_{t+1} ; \theta, \rho\right) \approx \omega^{0}(\theta, \rho)+\omega^{1}(\theta, \rho)\left(s_{t}-1\right)+\omega^{2}(\theta, \rho)\left(s_{t+1}-1\right)
$$

where $\omega^{0}(\theta, \rho)=\omega^{2}(\theta, \rho)=\varphi(1 ; \theta, \rho)[A+1-\delta]$ is an approximation to the mean growth rate, $\bar{\gamma}(\theta, \rho)$, and $\omega^{1}(\theta, \rho)=\varphi_{s}(1 ; \theta, \rho)(A+1-\delta)-\varphi(1 ; \theta, \rho)(1-\delta)$, where $\varphi_{s}(1 ; \theta, \rho)=\partial \varphi(s ; \theta, \rho) /\left.\partial s\right|_{s=1}$. It is more instructive to express these coefficients as

$$
\begin{aligned}
& \omega^{0}(\theta, \rho)=\omega^{2}(\theta, \rho)=\varphi(1 ; \theta, \rho)[A+1-\delta]=\bar{\gamma}(\theta, \rho) \\
& \omega^{1}(\theta, \rho)=\bar{\gamma}(\theta, \rho)(\varsigma-v)
\end{aligned}
$$

where $\varsigma \equiv \varphi_{s}(1 ; \theta, \rho) /\left.\varphi(s ; \theta, \rho)\right|_{s=1}$ is the elasticity of the (broad) savings rate with respect to the shock and $v$ is as before.

It is straightforward to compute the first-order autocorrelation coefficient for the growth rate, 
$\rho_{\gamma}$. It follows that

$$
\begin{aligned}
& \rho_{\gamma} \approx \rho+\omega^{1}(\theta, \rho) \omega^{2}(\theta, \rho) /\left[\left(\omega^{1}(\theta, \rho)\right)^{2}+\left(\omega^{2}(\theta, \rho)\right)^{2}+\rho \omega^{1}(\theta, \rho) \omega^{2}(\theta, \rho)\right] \\
& \rho_{\gamma} \approx \rho+(\varsigma-v) /\left[1+(\varsigma-v)^{2}+\rho(\varsigma-v)\right] .
\end{aligned}
$$

\section{Derivation of the First-Order Conditions for the Model of Section 4}

The Euler equations for an interior solution are given by

(B.1) $u_{c}(t)=E_{t}\left\{u_{c}(t+1)\left[1-\delta+F_{k}(t+1)\right]\right\}$

(B.2) $u_{c}(t)=E_{t}\left\{u_{c}(t+1)\left[1-\delta+n_{t+1} F_{z}(t+1)\right]\right\}$

where $u_{c}$ is the partial derivative of $u$ with respect to $c$, and $F_{k}$ and $F_{z}$ are the partial derivatives of $F$ with respect to capital and effective labor.

For the Cobb-Douglas form, (B.1) and (B.2) can be combined to yield

$$
E_{t}\left\{u_{c}(t+1)\left[\alpha F(t+1) / k_{t+1}-(1-\alpha) F(t+1) / h_{t+1}\right]\right\}=0
$$

It follows that in any interior equilibrium, we must have that $h_{t} / k_{t}=(1-\alpha) / \alpha$ for all $t$. This is an important property of the specification of a Cobb-Douglas production function with equal depreciation rates: the physical capital/human capital ratio is independent of the level of employment and the productivity shock.

Given this and setting $A^{*}=A(1-\alpha)^{1-\alpha} \alpha^{\alpha}$, it follows that

$$
c_{t}=k_{t}\left[s_{t} A^{*} n_{t}^{1-\alpha}\left(\left(1-n_{t}\right) / n_{t}\right)((1-\alpha) / \alpha \psi)\right] \equiv k_{t} g_{1}\left(s_{t}, n_{t}\right)
$$

Using this, we obtain

$$
k_{t+1}=k_{t}\left[s_{t} A^{*} n_{t}^{1-\alpha}\left(1-\frac{1-\alpha}{\psi} \frac{1-n_{t}}{n_{t}}\right)+1-\delta\right] \equiv k_{t} g_{2}\left(s_{t}, n_{t}\right)
$$

Finally, after substitution, the relevant Euler equation becomes 
(B.6) $\left[g_{1}\left(s_{t}, n_{t}\right)\left(1-n_{t}\right)^{\psi}\right]^{-\sigma}\left(1-n_{t}\right)^{\psi}=\beta \int_{s}\left[g_{2}\left(s_{t}, n_{t}\right) g_{1}\left(s_{t+1}, n_{t+1}\right)\left(1-n_{t+1}\right)^{\psi}\right]^{-\sigma}$

$\left.\times\left(1-n_{t+1}\right)^{\psi}\left[1-\delta+s_{t+1} A^{*}\left(n_{t+1}\right)^{1-\alpha}\right] P\left(s_{t}, d s_{t+1}\right)\right\}$.

A solution to this equation is a function $n^{*}: S \rightarrow[0,1]$ with $n_{t}=n^{*}\left(s_{t}\right)$. Note that given $n^{*}$, the optimal solution to the planner's problem is given by

(B.7) $n_{t}=n^{*}\left(s_{t}\right)$

$$
\begin{aligned}
& k_{t+1}=k_{t} g_{2}\left(s_{t}, n^{*}\left(s_{t}\right)\right) \\
& h_{t+1}=((1-\alpha) / \alpha) k_{t} g_{2}\left(s_{t}, n^{*}\left(s_{t}\right)\right) \\
& c_{t}=k_{t} g_{1}\left(s_{t}, n^{*}\left(s_{t}\right)\right)
\end{aligned}
$$

which correspond to the equations calculated in Section 4. 


\section{Appendix C: Basic Data From Simulations}

Table C.1. The Effect of $\zeta$ on $E(\gamma)$, Selected Values of $\sigma$

\begin{tabular}{|c|c|c|c|c|c|c|c|}
\hline Case & $\sigma$ & $\rho$ & $\zeta$ & $\sigma_{s}$ & $E(\gamma)$ & $\sigma_{\gamma}$ & $\rho_{\gamma}$ \\
\hline 1 & 1.0 & 0.9 & 0.095 & 0.126 & $2.48 \%$ & 0.108 & 0.013 \\
\hline 2 & 1.0 & 0.9 & 0.08 & 0.106 & $2.34 \%$ & 0.091 & 0.012 \\
\hline 3 & 1.0 & 0.9 & 0.06 & 0.080 & $2.38 \%$ & 0.066 & 0.045 \\
\hline 4 & 1.5 & 0.9 & 0.095 & 0.126 & $2.24 \%$ & 0.077 & -0.001 \\
\hline 5 & 1.5 & 0.9 & 0.08 & 0.106 & $2.17 \%$ & 0.064 & -0.002 \\
\hline 6 & 1.5 & 0.9 & 0.06 & 0.080 & $2.22 \%$ & 0.047 & 0.029 \\
\hline 7 & 1.5 & 0.8 & 0.14 & 0.135 & $2.80 \%$ & 0.120 & -0.096 \\
\hline 8 & 1.5 & 0.8 & 0.12 & 0.115 & $2.64 \%$ & 0.101 & -0.056 \\
\hline 9 & 1.5 & 0.8 & 0.095 & 0.091 & $2.30 \%$ & 0.080 & -0.082 \\
\hline 10 & 1.5 & 0.8 & 0.08 & 0.077 & $2.26 \%$ & 0.067 & -0.079 \\
\hline 11 & 1.5 & 0.8 & 0.06 & 0.058 & $2.09 \%$ & 0.051 & -0.071 \\
\hline 12 & 2.0 & 0.9 & 0.095 & 0.126 & $2.27 \%$ & 0.070 & -0.004 \\
\hline 13 & 2.0 & 0.9 & 0.08 & 0.106 & $2.19 \%$ & 0.059 & -0.005 \\
\hline 14 & 2.0 & 0.9 & 0.06 & 0.080 & $2.22 \%$ & 0.043 & 0.024 \\
\hline 15 & 2.5 & 0.9 & 0.095 & 0.126 & $2.32 \%$ & 0.067 & -0.006 \\
\hline 16 & 2.5 & 0.9 & 0.08 & 0.106 & $2.23 \%$ & 0.056 & -0.007 \\
\hline 17 & 2.5 & 0.9 & 0.06 & 0.080 & $2.23 \%$ & 0.041 & 0.022 \\
\hline $\mathrm{SH}$ mean & - & - & - & & $2.04 \%$ & 0.062 & 0.123 \\
\hline SH Q1 & - & - & - & & $0.91 \%$ & 0.041 & -0.039 \\
\hline SH Q3 & - & - & - & & $3.25 \%$ & 0.076 & 0.307 \\
\hline
\end{tabular}




\begin{tabular}{||c|c|c|c|c|c|c|c||}
\hline \multicolumn{7}{|c||}{ Table C.2. The Effect of $\rho, \sigma=1.5$} \\
\hline Case & $\sigma$ & $\rho$ & $\zeta$ & $E(\gamma)$ & $\sigma_{\gamma}$ & $\rho_{\gamma}$ & $\sigma_{s}$ \\
\hline 1 & 1.5 & 0.7 & 0.08 & $2.28 \%$ & 0.070 & -0.131 & 0.065 \\
\hline 2 & 1.5 & 0.8 & 0.08 & $2.26 \%$ & 0.067 & -0.079 & 0.077 \\
\hline 3 & 1.5 & 0.9 & 0.08 & $2.17 \%$ & 0.064 & -0.002 & 0.106 \\
\hline 4 & 1.5 & 0.915 & 0.08 & $1.94 \%$ & 0.065 & 0.004 & 0.115 \\
\hline SH mean & - & - & - & $2.04 \%$ & 0.062 & 0.123 & \\
\hline SH Q1 & - & - & - & $0.91 \%$ & 0.041 & -0.039 & \\
\hline SH Q3 & - & - & - & $3.25 \%$ & 0.076 & 0.307 & \\
\hline \hline
\end{tabular}

Table C.3. The Effect of $\gamma_{S S}$ on the Effects of Uncertainty, $\sigma=1.5$

\begin{tabular}{||c|c|c|c|c|c|c|c||}
\hline Case & $\gamma_{S S}$ & $\rho$ & $\zeta$ & $E(\gamma)$ & $\sigma_{\gamma}$ & $\rho_{\gamma}$ & $\sigma_{s}$ \\
\hline 1 & $0.0 \%$ & 0.9 & 0.095 & $0.26 \%$ & 0.078 & -0.023 & 0.126 \\
\hline 2 & $0.0 \%$ & 0.9 & 0.08 & $0.18 \%$ & 0.0649 & -0.023 & 0.106 \\
\hline 3 & $0.0 \%$ & 0.9 & 0.06 & $0.21 \%$ & 0.048 & 0.008 & 0.080 \\
\hline 4 & $2.0 \%$ & 0.9 & 0.095 & $2.27 \%$ & 0.077 & -0.001 & 0.126 \\
\hline 5 & $2.0 \%$ & 0.9 & 0.08 & $2.19 \%$ & 0.064 & -0.002 & 0.106 \\
\hline 6 & $2.0 \%$ & 0.9 & 0.06 & $2.22 \%$ & 0.047 & 0.029 & 0.080 \\
\hline 7 & $4.0 \%$ & 0.9 & 0.095 & $4.22 \%$ & 0.077 & 0.024 & 0.126 \\
\hline 8 & $4.0 \%$ & 0.9 & 0.08 & $4.16 \%$ & 0.064 & 0.021 & 0.106 \\
\hline 9 & $4.0 \%$ & 0.9 & 0.06 & $4.24 \%$ & 0.047 & 0.051 & 0.080 \\
\hline SH mean & - & - & - & $2.04 \%$ & 0.062 & 0.123 & \\
\hline SH Q1 & - & - & - & $0.91 \%$ & 0.041 & -0.039 & \\
\hline SH Q3 & - & - & - & $3.25 \%$ & 0.076 & 0.307 & \\
\hline \hline
\end{tabular}




\section{References}

Aizenman, J. and N. P. Marion. 1993. "Policy Uncertainty, Persistence and Growth.” Review of International Economics 1 (June): 145-163.

Alvarez, F. and N. L. Stokey. 1998. "Dynamic Programming with Homogeneous Functions.” Journal of Economic Theory 82 (September): 167-189.

Atkeson, A. and C. Phelan. 1994. "Reconsidering the Costs of Business Cycles with Incomplete Markets.” NBER Macreconomics Annual 1994, S. Fischer and J. J. Rotemberg, eds., pp. 187-207. Cambridge, Mass.: MIT Press.

Bean, C. R. 1990. "Endogenous Growth and the Procyclical Behaviour of Productivity." European Economic Review 34 (May): 355-363.

Brock, W. A. and L. J. Mirman. 1972. "Optimal Economic Growth and Uncertainty: The Discounted Case." Journal of Economic Theory 4: 497-513.

Burnside, C. and M. Eichenbaum. 1994. "Factor Hoarding and the Propagation of Business Cycle Shocks." Working Paper 4675, National Bureau of Economic Research.

Cogley, T. and J. M. Nason. 1995. "Output Dynamics in Real-Business-Cycle Models.” American Economic Review 85 (June): 492-511.

Cooley, T. F. (ed.) 1995. Frontiers of Business Cycle Research. Princeton, N.J.: Princeton University Press.

Cooley, T. F. and E. C. Prescott. 1995. "Economic Growth and Business Cycles." In T. F. Cooley, ed., Frontiers of Business Cycle Research, pp. 1-38. Princeton, N.J.: Princeton University Press.

de Hek, P. A. 1999. “On Endogenous Growth Under Uncertainty.” International Economic Review 40 (August): 727-744. 
Dotsey, M. and P.-D. Sarte. 1997. "Inflation Uncertainty and Growth in a Simple Monetary Model." Federal Reserve Bank of Richmond.

Eaton, J. 1981. "Fiscal Policy, Inflation and the Accumulation of Risky Capital." Review of Economic Studies 48 (July): 435-445.

Fatas, A. 2000. "Endogenous Growth and Stochastic Trends.” Journal of Monetary Economics 45 (February): 107-128.

Gomme, P. 1993. "Money and Growth Revisited: Measuring the Costs of Inflation in an Endogenous Growth Model.” Journal of Monetary Economics 32 (August): 51-77.

Hall, R. 1998. “Controlling the Price Level.” Working Paper 6914, National Bureau of Economic Research. Forthcoming in Proceedings of the Yale Conference on Irving Fisher.

Hopenhayn, H. A. and M. E. Muniagurria. 1996. "Policy Variability and Economic Growth.” Review of Economic Studies 63 (October): 611-625.

Imrohoroğlu, A. 1989. "Cost of Business Cycles with Indivisibilities and Liquidity Constraints." Journal of Political Economy 97 (December): 1364-1383.

Jones, L. E. and R. E. Manuelli. 1990. “A Convex Model of Equilibrium Growth: Theory and Policy Implications." Journal of Political Economy 98 (October, Part 1): 1008-1038.

Jones, L. E. and R. E. Manuelli. 1999. “The Equivalence Between Productivity and Tax Shocks.” Working Paper, University of Wisconsin.

Jones, L. E.; R. E. Manuelli; and P. E. Rossi. 1993. "Optimal Taxation in Models of Endogenous Growth.” Journal of Political Economy 101 (June): 485-517.

Jones, L. E.; R. E. Manuelli; H. Siu; and E. Stacchetti. 1998. “The Business Cycle Frequency Properties of Models of Endogenous Growth.” Working Paper. 
Judson, R. and A. Orphanides. 1996. "Inflation, Volatility and Growth," Finance and Economics Discussion Series 96-19, Board of Governors of the Federal Reserve System.

King, R. G.; C. I. Plosser; and S. T. Rebelo. 1988. "Production, Growth and Business Cycles: II. New Directions.” Journal of Monetary Economics 21 (March/May): 309-341.

King, R. G. and S. T. Rebelo. 1988. "Business Cycles with Endogenous Growth.” Working Paper, University of Rochester.

Kocherlakota, N. R. and K. M. Yi. 1994. "Is There Endogenous Long-Run Growth? Evidence From the United States and the United Kingdom.” Journal of Money, Credit, and Banking 29 (May): 235-262.

Kormendi, R. C. and P. G. Meguire. 1985. "Macroeconomic Determinants of Growth: CrossCountry Evidence.” Journal of Monetary Economics 16 (September): 141-163.

Levhari D. and T. N. Srinivasan. 1969. “Optimal Savings Under Uncertainty.” Review of Economic Studies 36 (April): 153-163.

Lucas, R. E., Jr. 1987. Models of Business Cycles. Yrjo Jahnsson Lectures Series. New York: Blackwell.

Lucas, R. E., Jr. 1988. “On the Mechanics of Economic Development.” Journal of Monetary Economics 22 (July): 3-42.

Manuelli, R. and T. J. Sargent. 1988. "Models of Business Cycles: A Review Essay.” Journal of Monetary Economics 22 (November): 523-542.

Obstfeld, M. 1994. "Risk-Taking, Global Diversification, and Growth.” American Economic Review 84 (December): 1310-1329.

Phelps, E. S. 1962. "The Accumulation of Risky Capital: A Sequential Utility Analysis." Econometrica 30 (October): 729-743. 
Ramey, G. and V. A. Ramey. 1995. "Cross-Country Evidence on the Link Between Volatility and Growth.” American Economic Review 85 (December): 1138-1151.

Romer, P. 1986. "Increasing Returns and Long Run Growth." Journal of Political Economy 94: $1002-1037$.

Rothschild, M. and J. E. Stiglitz. 1971. "Increasing Risk II: Its Economic Consequences.” Journal of Economic Theory 3 (March): 66-84.

Rudin, W. 1974. Real and Complex Analysis. New York: McGraw Hill.

Stokey, N. L. and R. E. Lucas, Jr. (with E. C. Prescott). 1989. Recursive Methods in Economic Dynamics. Cambridge, Mass: Harvard University Press.

Summers, R. and A. Heston. 1991. "The Penn World Table (Mark 5): An Expanded Set of International Comparisons, 1950-1988." Quarterly Journal of Economics 106 (May): 327-368.

Summers, R. and A. Heston. 1993. "Penn World Tables, Version 5.5," available on diskette from the National Bureau of Economic Research. 
Figure 1: Curvature and Mean Growth Rates

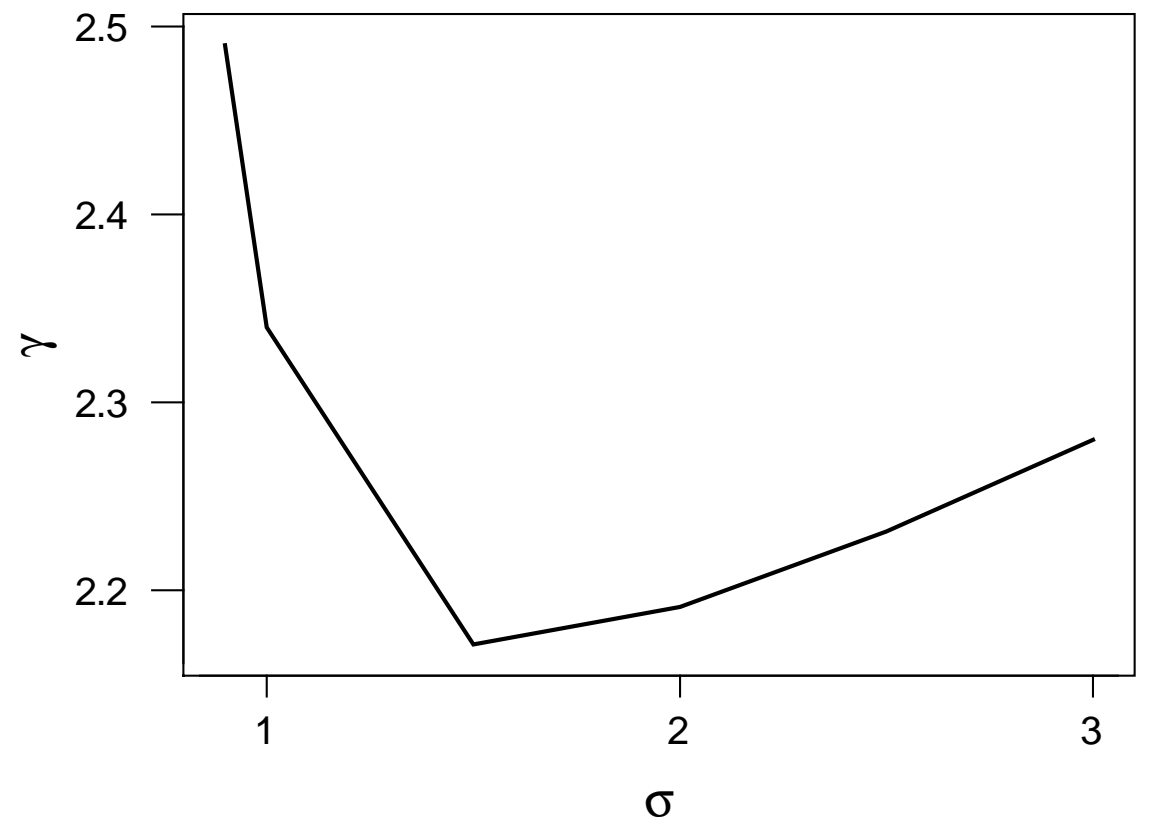


Figure 2: Standard Deviation of Innovations and Mean Growth

Solid - correlation $=.8$, Dashed - correlation $=.9$

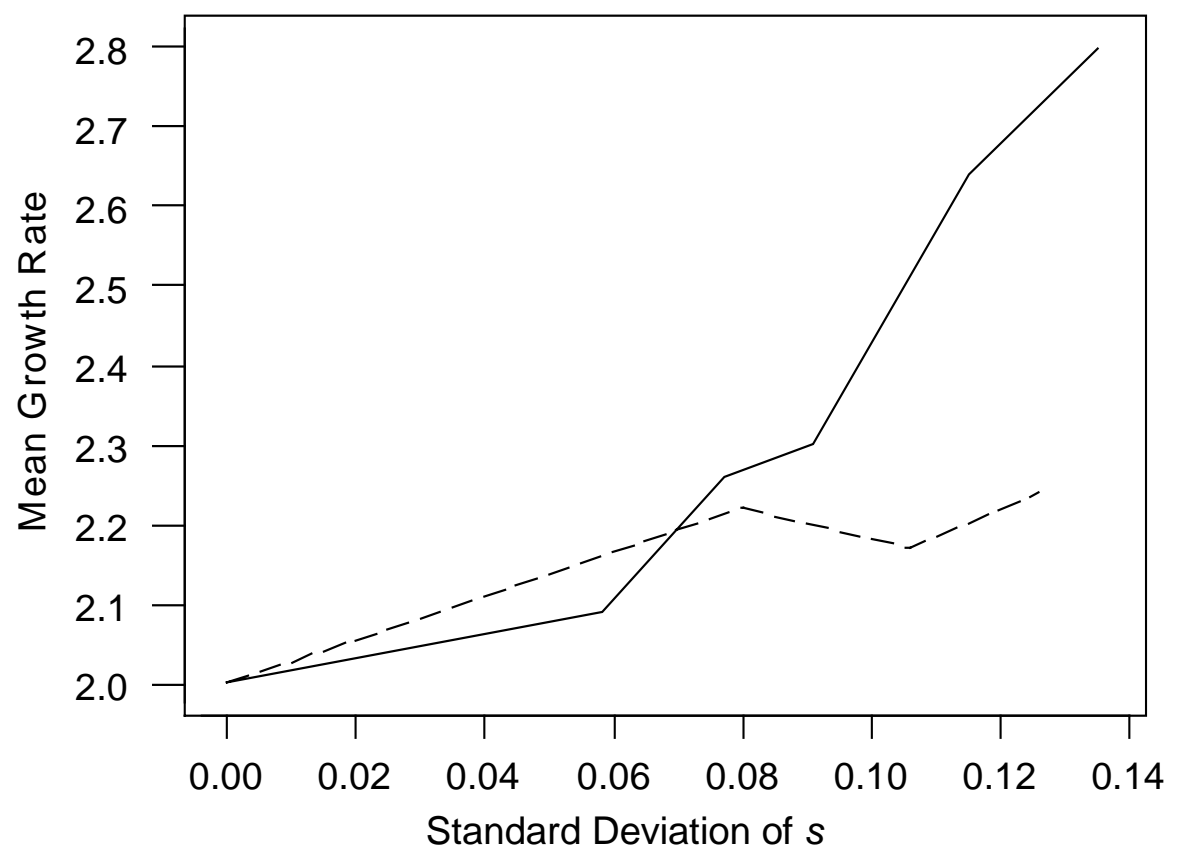




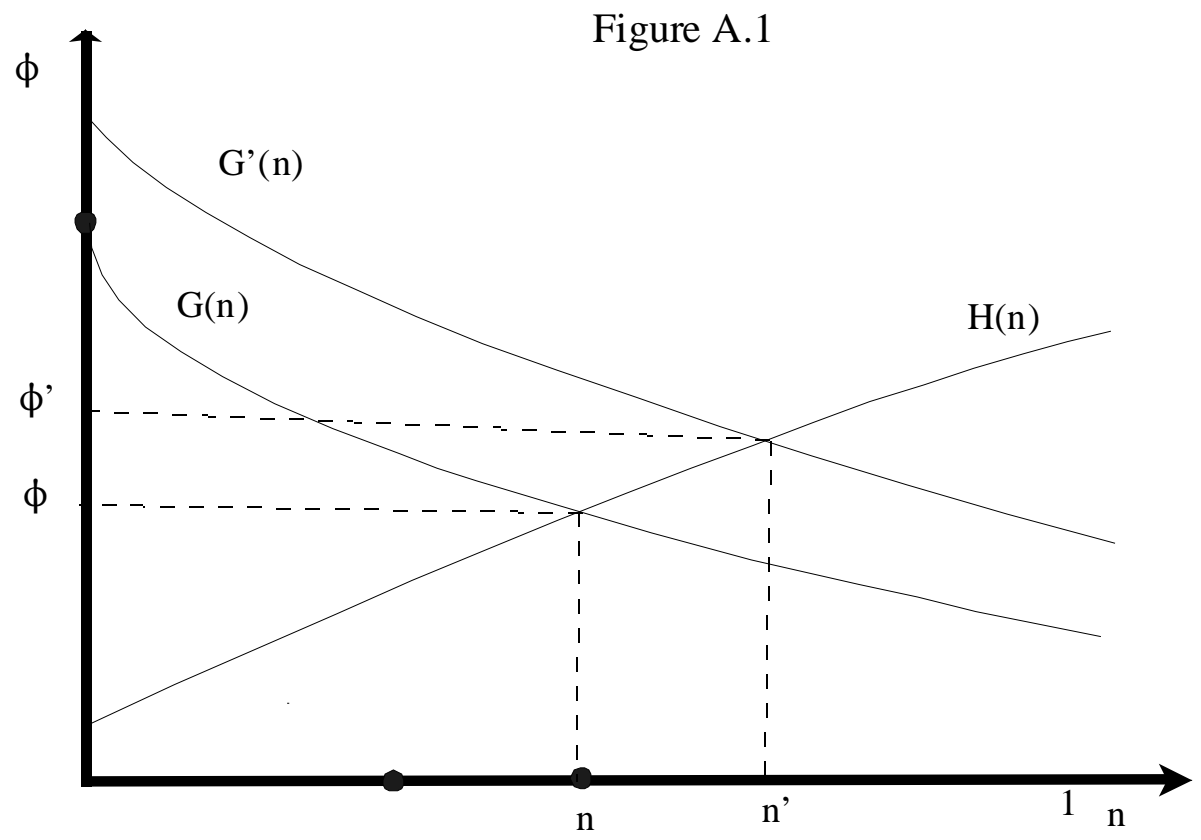




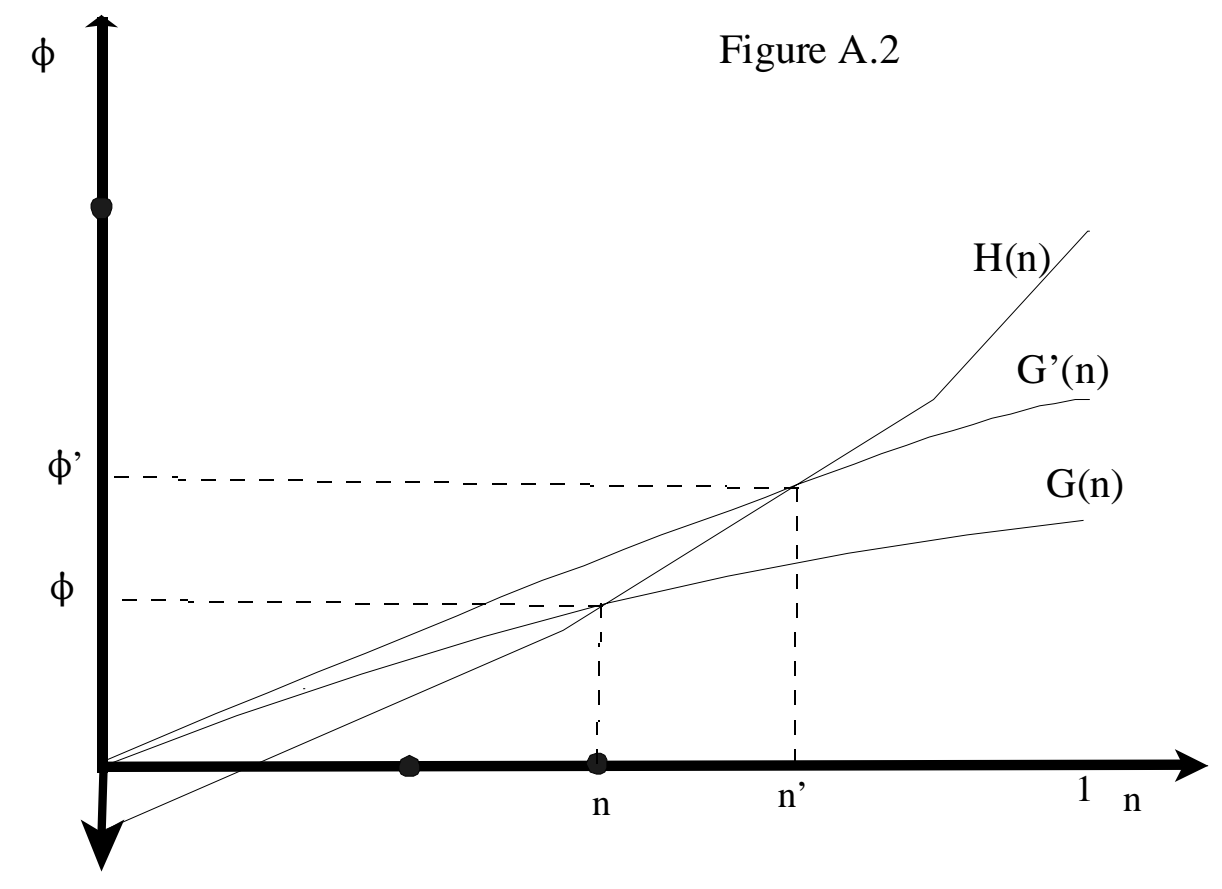

